November 10, 2018

\title{
The Rest-Frame Instant Form and Dirac Observables for the Open Nambu String
}

\author{
David Alba \\ Dipartimento di Fisica \\ Universita' di Firenze \\ Polo Scientifico, via Sansone 1 \\ 50019 Sesto Fiorentino, Italy \\ E-mail ALBA@FI.INFN.IT \\ Horace W. Crater \\ The University of Tennessee Space Institute \\ Tullahoma, TN 37388 USA \\ E-mail: hcrater@utsi.edu \\ Luca Lusanna \\ Sezione INFN di Firenze \\ Polo Scientifico \\ Via Sansone 1 \\ 50019 Sesto Fiorentino (FI), Italy \\ E-mail: lusanna@fi.infn.it
}

\begin{abstract}
The rest-frame instant form of the positive-energy part of the open Nambu string is developed. The string is described as a decoupled non-local canonical non-covariant Newton-Wigner center of mass plus a canonical basis of Wigner-covariant relative variables living in the Wigner 3 -spaces. The center of mass carries a realization of the Poincare' algebra depending upon the invariant mass and the rest-spin of the string, functions of the relative variables. A canonical basis of gauge invariant Dirac observables is built with Frenet-Serret geometrical methods. Some comments on canonical quantization are made.
\end{abstract}




\section{INTRODUCTION}

The recently developed parametrized Minkowski theories [1-3] allow one to give a unified description of isolated relativistic systems (particles, strings, fluids, fields) admitting a Lagrangian description both in inertial and non-inertial frames of Minkowski space-time. A basic tool is the family of admissible $3+1$ splittings of Minkowski space-time [3] (i.e. a convention for clock synchronization identifying the instantaneous 3-spaces [2]) and the use of radar 4-coordinates adapted to a time-like observer. In this way it is possible to show that the transition from a non-inertial frame to every other frame is a gauge transformation: as a consequence special relativistic physics is not influenced by the choice of the clock synchronization convention and by the choice of the 3-coordinates on the instantaneous 3-space (only the appearance of the phenomena is changed).

In particular we can define the inertial rest-frame instant form of dynamics of every isolated system by considering a $3+1$ splitting whose instantaneous 3 -spaces are orthogonal to the conserved 4-momentum of the isolated system: it corresponds to the intrinsic inertial rest frame centered on an inertial observer ${ }^{1}$. In this formulation there is a complete understanding and classification [4,5] of the collective relativistic 4-variables (canonical non-covariant Newton-Wigner center of mass, non-canonical covariant Fokker-Price center of inertia, noncanonical non-covariant Møller center of energy) for any isolated system. They can be built by using only the ten Poincare' generators of the isolated system (since the generators are non-local quantities knowing the whole instantaneous 3-space, also the collective variables are non-local quantities). It turns out that every isolated system can be described as a decoupled non-covariant point particle (corresponding to the Newton-Wigner center of mass) carrying a pole-dipole structure, i.e. the invariant mass $M$ and the rest spin $\overrightarrow{\vec{S}}$ of the system. Associated with this non-covariant non-local ${ }^{2}$ particle there is an external realization of the Poincare' algebra (its Lorentz boosts induce Wigner rotations on Wigner spin-1 3-vectors inside the instantaneous Euclidean 3-spaces, named Wigner 3-spaces). Inside these Wigner 3 -spaces the dynamics of the isolated system is described by Wigner-covariant relative variables. The quantities $M$ and $\vec{S}$ are functions only of these relative variables and moreover they are the energy and the angular momentum of a unfaithful inner realization of the Poincare' algebra, whose generators are determined by the energy-momentum tensor of the system. It is unfaithful because the inner 3-momentum vanishes: this is the rest-frame condition identifying the rest frame. The (interaction-dependent) inner Lorentz boosts depend on the inner 3-center of mass, canonically conjugate to the vanishing inner 3-momentum, inside the 3-space. In order not to have a double counting of the center of mass (external, inner) we must eliminate this variable by means of a statement on the inner Lorentz boosts. If we ask for the vanishing of the inner Lorentz boost, it turns out that the inner center of mass is at the origin of each instantaneous 3-space and that the world-line of the inertial observer on which the rest frame is centered is the Fokker-Price covariant center of inertia.

Previously we have done a detailed study of the rest-frame instant form of the isolated system composed of $\mathrm{N}$ positive-energy charged scalar particles plus the electro-magnetic field

\footnotetext{
${ }^{1}$ See Ref.[3] for the non-inertial rest frames and for the associated non-inertial rest-frame instant form of dynamics.

${ }^{2}$ No one can observe it, so that its non-covariance is irrelevant. It is a notion like the wave function of the universe.
} 
[4-6]. In this paper we want to extend this study to the open Nambu string [7] (the closed one could also be studied with these methods). As a byproduct of this description we will be able to find a complete canonical set of classical Dirac observables for the Nambu string, a result which was till now missing (see Ref.[8] for previous attempts).

As a consequence, the final description of the open Nambu string consists of a decoupled non-covariant canonical center of mass carrying an invariant mass $M$ and a rest-frame spin $\vec{S}$ functions only of the Dirac observables. The final Poincare' algebra is the external one of a point-like particle: it depends upon $M$ and $\vec{S}$, whose Poisson brackets are $\left\{M, \bar{S}^{i}\right\}=0$ and $\left\{\bar{S}^{i}, \bar{S}^{j}\right\}=\epsilon^{i j k} \bar{S}^{k}$. In terms of the canonical basis of Dirac observables we have a non-linear realization of an $\mathrm{O}(3)$-algebra with generators $\bar{S}^{i}$, like in a 3-dimensional $\sigma$-model, plus a $\mathrm{O}(3)$-scalar invariant mass $M$ (a non-linear functional of the Dirac observables). Therefore we have an implementation of the old approach of Ref.[9] in terms of the rest-frame instant form of dynamics, in which the world-sheet 4-coordinates are derived quantities (in general they are not canonical quantities, but the analogue of predictive coordinates as shown in Ref.[4], where one can find the relevant bibliography).

In Section II we review the standard action of the open Nambu string and some technical points connected with the fact that its endpoints move along null curves in Minkowski spacetime, so that the coordinates of the orthogonal gauge (OG) are singular there [10]. Then we review the reformulation of the open string given in Ref. [11] (see Ref.[12] for the closed string)with the needed distributions, whose expression can be found in Appendix A.

In Section III we reformulate the open string as a reparametrized Minkowski theory, which allows to describe the part of the spectrum with positive energy. Only the transversality first class constraints are present in this formulation, because the mass shell constraints are absent due to the different canonical variables, parametrizing the string, introduced after an admissible $3+1$ splitting of Minkowski space-time.

Then in Section IV we define the rest-frame instant form of the positive energy open string.

In Section V we identify a Frenet-Serret (FS) canonical basis adapted to the transversality constraints by means of a Shanmugadhasan canonical transformation. The Dirac observables turn out to be action-angle variables. Also a set of Lorentz-scalar Dirac observables, not depending on angles but on the FS curvature and torsion of the open string, is identified, but the associated canonical basis is not explicitly known.

In Section VI we show how to replace the action-angle variables with oscillators.

In the Conclusions, after some comments on previous attempts to find Dirac observables of the string and a consistent quantization in $D=4$ dimensions, we discuss which are the problems one will face in attempting to quantize the Dirac observables of a FS canonical basis. Even if the quantization problem is still completely open ${ }^{3}$, the classical geometrical problems are finally understood.

\footnotetext{
${ }^{3}$ In general the quantization of the reduced phase space resulting from the solution of the constraints is inequivalent from the quantization of the original phase space and of the constraints followed by a quantum reduction to the physical Hilbert space.
} 


\section{A REVIEW OF THE NAMBU ACTION OF THE OPEN STRINGS AND OF THE PROBLEMS CONNECTED WITH THE END POINTS}

In this Section we discuss the kinematical problems arising from the fact that the end points of the open string move on null surfaces. These items are usually ignored, but they are relevant for the choice of the symplectic structure in phase space.

Let us consider the action for the open Nambu string [7] $(\hbar=c=1$; the metric is $\left.\eta_{\mu \nu}=(+---)\right)$ in the notation of Ref.[10] (see also Refs. $[8,11,12]$ )

$$
S=-N \int_{\tau_{1}}^{\tau_{2}} d \tau \int_{0}^{\pi} d \lambda \sqrt{-h(\tau, \lambda)}, \quad L=-N \sqrt{-h},
$$

where $N=\frac{1}{2 \pi \alpha^{\prime}}, \dot{x}^{\mu}(\tau, \lambda)=\partial x^{\mu}(\tau, \lambda) / \partial \tau, x^{\prime \mu}(\tau, \lambda)=\partial x^{\mu}(\tau, \lambda) / \partial \lambda$, and

$$
\begin{aligned}
-h & =-\operatorname{det}\left\|h_{\alpha \beta}\right\|=\left(\dot{x} \cdot x^{\prime}\right) 2-\dot{x}^{2} x^{\prime 2} \geq 0, \\
\left\|h_{\alpha \beta}\right\| & =\left\|\partial_{\alpha} x^{\mu} \partial_{\beta} x_{\mu}\right\|=\left(\begin{array}{cc}
\dot{x}^{2} & \dot{x} \cdot x^{\prime} \\
\dot{x} \cdot x^{\prime} & x^{\prime 2}
\end{array}\right), \quad \alpha=0,1, \quad \partial_{0}=\frac{\partial}{\partial \tau}, \quad \partial_{1}=\frac{\partial}{\partial \lambda}, \\
\left\|h^{\alpha \beta}\right\| & =\frac{1}{h}\left(\begin{array}{cc}
x^{\prime 2} & -\left(\dot{x} \cdot x^{\prime}\right) \\
-\left(\dot{x} \cdot x^{\prime}\right) & \dot{x}^{2}
\end{array}\right) .
\end{aligned}
$$

The condition $-h \geq 0$ means that the surface swept by the string in the space-time is everywhere time-like or null (i.e. it is a causal surface). The strip $0<\lambda<\pi$ is mapped in the world-sheet spanned by the string in Minkowski space, which is described by the coordinates $x^{\mu}(\tau, \lambda)$ in an arbitrary inertial frame.

Let us define the following two quantities

$$
\begin{aligned}
& P^{\mu}(\tau, \lambda)=-\frac{\partial L}{\partial \dot{x}_{\mu}(\tau, \lambda)}=N \sqrt{-h} h^{0 \alpha} \partial_{\alpha} x^{\mu}=\frac{N}{\sqrt{-h}}\left(\left(\dot{x} \cdot x^{\prime}\right) x^{\prime \mu}-{x^{\prime}}^{2} \dot{x}^{\mu}\right), \\
& \Pi^{\mu}(\tau, \lambda)=-\frac{\partial L}{\partial x^{\prime}{ }_{\mu}(\tau, \lambda)}=N \sqrt{-h} h^{1 \alpha} \partial_{\alpha} x^{\mu}=\frac{N}{\sqrt{-h}}\left(\left(\dot{x} \cdot x^{\prime}\right) \dot{x}^{\mu}-\dot{x}^{2} x^{\prime \mu}\right),
\end{aligned}
$$

where $P^{\mu}$ is the canonical momentum, which satisfies the identities

$$
\begin{aligned}
& P^{2}(\tau, \lambda)+N^{2} x^{\prime 2}(\tau, \lambda)=0, \quad P(\tau, \lambda) \cdot x^{\prime}(\tau, \lambda)=0, \quad \Pi^{2}(\tau, \lambda)+N^{2} \dot{x}^{2}(\tau, \lambda)=0, \\
& \Pi(\tau, \lambda) \cdot \dot{x}(\tau, \lambda)=0, \quad \Pi(\tau, \lambda) \cdot x^{\prime}(\tau, \lambda)=N \sqrt{-h(\tau, \lambda)}, \\
& \Pi(\tau, \lambda) \cdot P(\tau, \lambda)=N^{2} \dot{x}(\tau, \lambda) \cdot x^{\prime}(\tau, \lambda), \quad P(\tau, \lambda) \cdot \dot{x}(\tau, \lambda)=N \sqrt{-h(\tau, \lambda)} .
\end{aligned}
$$

At the Hamiltonian level we get the usual first class constraints $P^{2}(\tau, \lambda)+N^{2} x^{\prime 2}(\tau, \lambda) \approx 0$ and $P(\tau, \lambda) \cdot x^{\prime}(\tau, \lambda) \approx 0$.

The Hessian matrix is [10] 


$$
\begin{aligned}
\left\|W^{\mu \nu}(\tau, \lambda)\right\| & =\left\|\frac{\partial^{2} L}{\partial \dot{x}_{\mu} \partial \dot{x}_{\nu}}\right\|= \\
& =\left\|\frac{N x^{\prime 2}}{(-h)^{\frac{3}{2}}}\left[-h \eta^{\mu \nu}+{x^{\prime}}^{2} \dot{x}^{\mu} \dot{x}^{\nu}+\dot{x}^{2} x^{\prime \mu} x^{\prime \nu}-\left(\dot{x} \cdot x^{\prime}\right)\left(\dot{x}^{\mu} x^{\prime \nu}+x^{\prime \mu} \dot{x}^{\nu}\right)\right]\right\| .
\end{aligned}
$$

The 4-vectors $\dot{x}^{\mu}(\tau, \lambda)$ and $x^{\prime \mu}(\tau, \lambda)$ are the null eigenvectors of the Hessian matrix for every value of $\lambda$ except the end values $\lambda=0, \pi^{4}$. The non-null eigenvalues are degenerate for $\lambda \neq 0, \pi$, and are equal to $\frac{N x^{\prime 2}(\tau, \lambda)}{\sqrt{-h(\tau, \lambda)}}$. The non-null eigenvectors $\zeta_{\epsilon}^{\mu}(\tau, \lambda)$, with $\epsilon=1,2$, are orthogonal to $\dot{x}^{\mu}$ and $x^{\prime \mu}$, i.e. to the world-sheet, and so they are space-like.

The variational principle for the action $(2.1)$, with the variations $\delta_{0} x^{\mu}(\tau, \lambda)$ vanishing at $\tau=\tau_{1}, \tau_{2}$, is

$$
\delta S=\int_{\tau_{1}}^{\tau_{2}} d \tau \int_{0}^{\pi} d \lambda L_{\mu} \delta_{0} x^{\mu}-\left.\int_{\tau_{1}}^{\tau_{2}} d \tau \Pi_{\mu} \delta_{0} x^{\mu}\right|_{0} ^{\pi}=0
$$

and gives the following Euler-Lagrange equations and boundary conditions $(\stackrel{\circ}{=}$ means evaluated on the solutions)

$$
\begin{aligned}
L^{\mu}(\tau, \lambda) & =\dot{P}^{\mu}+\Pi^{\prime \mu}=-W^{\mu \nu}\left[\ddot{x}_{\nu}+\frac{1}{x^{\prime 2}}\left(\dot{x}^{2} x^{\prime \prime}{ }_{\nu}-2\left(\dot{x} \cdot x^{\prime}\right) \dot{x}_{\nu}^{\prime}\right)\right]= \\
& =N \partial_{\alpha}\left(\sqrt{-h} h^{\alpha \beta} \partial_{\beta} x^{\mu}\right) \stackrel{\circ}{=} 0, \\
\left.\Pi_{\mu} \delta_{0} x^{\mu}\right|_{0} ^{\pi} & =0 .
\end{aligned}
$$

Only two of the equations (2.7) are independent, for $\lambda \neq 0, \pi$, since we have the Noether identities $\dot{x}_{\mu} L^{\mu} \equiv 0$ and $x^{\prime}{ }_{\mu} L^{\mu} \equiv 0$. The boundary conditions in Eqs.(2.7) have been studied in detail in Refs. $[13,14]$, where it is shown that in regular coordinates, corresponding to a parametrization of the string world-sheet such that the tangent vectors $x^{\prime \mu}(\tau, \lambda)$ and $\dot{x}^{\mu}(\tau, \lambda)$ do not vanish and are independent, it amounts in requiring

$$
\left.\Pi_{\mu} x^{\prime \mu}\right|_{0} ^{\pi}=\left.N \sqrt{-h}\right|_{0} ^{\pi}=0
$$

4 The existence of the two null eigenvalues, and of the first class constraints, is connected to the $\tau, \lambda$ reparametrization invariance of the action; that is the action is invariant under the following transformations

$$
\begin{aligned}
\delta \tau & =\tilde{\tau}(\tau, \lambda)-\tau, \quad \delta \lambda=\tilde{\lambda}(\tau, \lambda)-\lambda, \quad \tilde{\lambda}(\tau, \overrightarrow{0})=0, \quad \tilde{\lambda}(\tau, \pi)=\pi, \\
\delta x^{\mu}(\tau, \lambda) & =\tilde{x}^{\mu}(\tilde{\tau}, \tilde{\lambda})-x^{\mu}(\tau, \lambda)=\delta_{0} x^{\mu}(\tau, \lambda)+\dot{x}^{\mu}(\tau, \lambda) \delta \tau+x^{\prime \mu}(\tau, \lambda) \delta \lambda=0,
\end{aligned}
$$

where the last formula expresses the fact that $x^{\mu}(\tau, \lambda)$ is scalar under reparametrization. 
As stressed by the authors of Refs.[13, 14], the requirement of regular coordinates is crucial for a consistent action principle. The restriction from (2.10) to (2.11) is due to the requirement that a variation of the boundaries must not violate the condition $-h \geq 0$.

Instead in the frequently used orthogonal gauge (OG) is defined by a choice of parameters which satisfy, besides $-h(\tau, \lambda) \geq 0$, the conditions

$$
\begin{aligned}
\dot{x}^{2}+x^{\prime 2}= & \left(\dot{x} \cdot x^{\prime}\right)=0, \quad \Rightarrow h_{\alpha \beta}=\dot{x}^{2}\left(\begin{array}{cc}
1 & 0 \\
0 & -1
\end{array}\right), \quad \text { with } \quad \dot{x}^{2} \geq 0 . \\
& \Downarrow \\
L^{\mu}= & N\left(\ddot{x}^{\mu}-x^{\prime \mu}\right) \stackrel{\circ}{=} 0, \quad P^{\mu}=N \dot{x}^{\mu}, \quad \Pi^{\mu}=-N x^{\prime \mu}, \\
W^{\mu \nu}= & -\frac{N}{\dot{x}^{2}}\left(\dot{x}^{2} \eta^{\mu \nu}-\dot{x}^{\mu} \dot{x}^{\nu}+x^{\prime \mu} x^{\prime \nu}\right) .
\end{aligned}
$$

At the end points the usual conditions of the OG gauge are

$$
x^{\prime \mu}(\tau, 0)=x^{\prime \mu}(\tau, \pi)=0, \quad \Rightarrow \quad \dot{x}^{2}(\tau, 0)=\dot{x}^{2}(\tau, \pi)=0,
$$

This in particular means that at the end points the induced metric $h_{\alpha \beta}$ has zero rank.

Let us remark that if one chooses coordinates such that

$$
\dot{x}^{2}(\tau, \lambda) \geq 0, \quad x^{\prime 2}(\tau, \lambda) \leq 0,
$$

then the condition $h=0$ implies two possible situations at the end points:

i) $x^{\prime 2}<0, \dot{x}^{2}=0,\left(\dot{x} \cdot x^{\prime}\right)=0$, with $\dot{x}^{\mu}$ and $x^{\prime \mu}$ independent and $\dot{x}^{\mu} \neq 0$. This is a regular case (the Jacobian of the map $(\tau, \lambda) \rightarrow x^{\mu}$ has maximal rank 2). In this case the rank of the induced metric $\left\|h_{\alpha \beta}\right\|$ is 1 , and the end points of the string describe null surfaces [13]. There is the possible case $\dot{x}^{\mu}=0$, which is a singular case (the Jacobian of the map $(\tau, \lambda) \rightarrow x^{\mu}$ has rank 1$)$.

ii) $x^{\prime 2}=0, \dot{x}^{2}=0,\left(\dot{x} \cdot x^{\prime}\right)=0$, with $x^{\prime \mu}$ collinear to $\dot{x}^{\mu}$. This is a singular case (where we may have $\Pi^{\mu} \neq 0$ as well as $\Pi^{\mu}=0$ ). The case $x^{\prime \mu}=0$, corresponding to the OG, may be considered as a particular case of (ii): OG is a singular case.

As a consequence, to describe the solutions of the classical equations of motion in a class of gauges including as a special case the OG, we have to work with the class (ii), that is necessarily with singular coordinates.

To check whether the boundary condition $\left.h\right|_{0} ^{\pi}=0$ is preserved in a singular case (since it was deduced in the regular one), one may perform a transformation from regular coordinates to those which will become singular at the end points, in the interior of the interval $(0, \pi)$, that is from the class (i) to the class (ii). As shown in Ref.[14], and more explicitly in Section I1 of Appendix I of Ref.[10], the Jacobian of the transformation vanishes as $\sqrt{\lambda}$ in $\lambda=0$ (and in an analogous way in $\pm \pi$ ), so ensuring, a fortiori, the vanishing of the new $h$ at the end points. 
So we will assume the boundary conditions (2.11), with a choice of coordinates falling into class (ii). In order to completely define the physical hypotheses, we will assume that the total momentum of the string $P^{\mu}$ be such that $P^{2} \geq 0$, with $P^{\mu} \neq 0$. As shown in Section I2 Appendix I of Ref.[10], this ensures a unique solution at the end points of the string with $P^{\mu}$ and $\Pi^{\mu}$ finite.

The previous discussion and the boundary conditions (2.8) suggest the usefulness of the following extension from the interval $(0, \pi)$ to $(-\pi, \pi)$

$$
x^{\mu}(\tau, \lambda)=x^{\mu}(\tau,-\lambda),
$$

and to the real line with $2 \pi$ periodicity. Let us stress that, with this kind of boundary conditions, the function $x^{\prime \mu}(\tau, \lambda)$, extended to all the real axis, may be discontinuous in $\lambda=0, \pi$.

The solutions of Eqs. (2.9) satisfying Eqs.(2.10) are

$$
\begin{aligned}
x^{\mu}(\tau, \lambda) & =q^{\mu}+\frac{P^{\mu}}{\pi N} \tau+f^{\mu}(\tau+\lambda)+f^{\mu}(\tau-\lambda)= \\
& =q^{\mu}+\frac{P^{\mu}}{\pi N} \tau+\frac{i}{\sqrt{\pi N}} \sum_{n \neq 0} \alpha_{n}^{\mu} \exp (-i n \tau) \cos n \lambda= \\
& =\frac{1}{2}\left[Q^{\mu}(\tau+\lambda)+Q^{\mu}(\tau-\lambda)\right], \\
\text { with } & \left(\frac{P^{\mu}}{2 \pi N}+\frac{d f^{\mu}(u)}{d u}\right)^{2}=0, \quad f^{\mu}(u)=f^{\mu}(u+2 n \pi),
\end{aligned}
$$

where $u=\tau \pm \lambda$, and where $P^{\mu}=\int_{0}^{\pi} d \sigma P^{\mu}(\tau, \lambda)$ is the conserved total momentum. The last of Eqs. (2.13) is a consequence of the OG conditions, and

$$
Q^{\mu}(\tau)=x^{\mu}(\tau, 0)=q^{\mu}+\frac{P^{\mu}}{\pi N} \tau+2 f^{\mu}(\tau), \quad Q^{\mu}(\tau+2 \pi)=Q^{\mu}(\tau)+2 \frac{P^{\mu}}{N},
$$

is the coordinate of the end point at $\lambda=0$. In terms of $Q^{\mu}$ Eqs.(2.13) imply $\frac{1}{4} \dot{Q}^{2}(\tau)=0$. The coordinates of the other end point are

$$
x^{\mu}(\tau, \pi)=x^{\mu}(\tau+\pi, 0)-\frac{P^{\mu}}{N}=Q^{\mu}(\tau+\pi)-\frac{P^{\mu}}{N} .
$$

In Ref.[15] it is shown that the end points suffer a constant translation of $\frac{2 P^{\mu}}{N}$ for every $\Delta \tau=$ $2 \pi$, and that for $\Delta \tau=\pi$ the distance between them is $\frac{P^{\mu}}{N}$. Their motion is given by a double helix with these periods. $Q^{\mu}(\tau)$ is a relevant function, because the transverse conformal invariant oscillators defined in Ref. [16] $\mathbf{A}_{n}=\sqrt{\frac{N}{2 \pi}} \int_{-\pi}^{\pi} d \rho \frac{d \mathbf{Q}(\rho)}{d \rho} \exp \left(i \pi n \frac{Q^{+}(\rho)}{P^{+}}\right)$, and the vertex of the dual models $\exp \left(i Q^{+}(z)\right)$ are defined in terms of it [17]. The Cauchy problem for the equations (2.9) is defined in Ref.[18]. 
The residual invariance group in the $\mathrm{OG}$ is given by the conformal transformations holding the end points $\lambda=0, \pi$ fixed:

$$
\begin{aligned}
& \tilde{\tau}=\tau_{1}+\tau+g(\tau+\lambda)+g(\tau-\lambda)=\tau_{1}+\tau+\sum_{n \neq 0} a_{n} \cos n \lambda \exp (-i n \tau), \\
& \tilde{\lambda}=\lambda+g(\tau+\lambda)-g(\tau-\lambda)=\lambda-i \sum_{n \neq 0} a_{n} \sin n \lambda \exp (-i n \tau),
\end{aligned}
$$

and the Jacobian is

$$
J=\frac{\partial(\tilde{\tau}, \tilde{\lambda})}{\partial(\tau, \lambda)}=\left[1+2 g^{\prime}(\tau+\lambda)\right]\left[1+2 g^{\prime}(\tau-\lambda)\right] \neq 0
$$

or $1+2 \frac{d g(u)}{d u} \neq 0$. These transformations leave invariant in form the wave equation in Eqs.(2.9) and the conditions (2.10).

To completely fix the gauge one has to add a further condition, for instance of the kind $[19]$

$$
t_{\mu}\left[x^{\mu}(\tau, \lambda)-q^{\mu}-\frac{P^{\mu} \tau}{\pi N}\right]=0,
$$

where $t_{\mu}$ is a constant vector. In the usual light-cone gauge one has $t^{\mu}=(1 ; 0,0,1)$, so that we have [compare with Eq. (2.13)]

$$
x^{+}(\tau, \lambda)-q^{+}-\frac{P^{+} \tau}{\pi N}=0, \quad A^{+}=A^{o}+A^{3}, \quad \text { implying } \quad f^{+}(u)=0 .
$$

In Ref.[20] it was noticed that, while in a time-like gauge, $t^{\mu}=(1 ; \mathbf{0})$ and $f^{o}(u)=0$, every solution of Eq. (2.9) can be made to satisfy equation (2.18) because $P^{0} \neq 0$, in the light-cone gauge solutions exist which cannot satisfy equation (2.18). See the discussion in Ref.[10] on how to recover these solutions in the Hamiltonian approach by introducing the following Poisson structure.

As a consequence of the previous discussion, in order to define a Poisson structure in the phase-space in Refs. $[8,10,11]$ the following extension of the coordinates outside the interval $(0, \pi)$ was used

$$
\begin{aligned}
x^{\mu}(\tau, \lambda) & =x^{\mu}(\tau,-\lambda,)=x^{\mu}(\tau, \lambda+2 n \pi), \\
P^{\mu}(\tau, \lambda) & =P^{\mu}(\tau,-\lambda)=P^{\mu}(\tau, \lambda+2 n \pi),
\end{aligned}
$$

where $\mathrm{n}$ is an integer, with the points $\lambda=2 n \pi$, for any $\mathrm{n}($ or $\lambda=(2 n+1) \pi$, for any $\mathrm{n})$, corresponding to the boundary values $x^{\mu}(\tau, 0)$ and $P^{\mu}(\tau, 0)\left(\right.$ or $x^{\mu}(\tau, \pi)$ and $\left.P^{\mu}(\tau, \pi)\right)$ as limit values from the open interval $(0, \pi)$.

With the kind of chosen boundary conditions and with this kind of extension to the real axis, the functions $x^{\prime \mu}(\tau, \lambda)$ and $P^{\prime \mu}(\tau, \lambda)$ will have in $\lambda_{i}\left(i=1,2 ; \lambda_{1}=0, \lambda_{2}=\pi\right)$ a jump, not present in the OG. This means that we must define the physical values of these functions 
as limit value from the open interval $(0, \pi)$, since the Fourier series will converge point-wise to the mean value of the left and right limits at the end points, that is to unphysical values.

Following Ref.[19] we introduce the following Poisson structure

$$
\left\{x^{\mu}(\tau, \lambda), P^{\nu}\left(\tau, \lambda^{\prime}\right)\right\}=-\eta^{\mu \nu} \Delta_{+}\left(\lambda, \lambda^{\prime}\right) \quad \longrightarrow \quad-\eta^{\mu \nu} \delta\left(\lambda-\lambda^{\prime}\right), \quad \text { for } \lambda, \lambda^{\prime} \in(0, \pi),
$$

where $\Delta_{+}\left(\lambda, \lambda^{\prime}\right)$ is an even delta function with period $2 \pi$, defined in Eqs.(A1) and (A3) of Appendix A.

This definition implies a suitable definition of the functional derivative

$$
\frac{\delta x_{\mu}(\lambda)}{\delta x^{\nu}\left(\lambda^{\prime}\right)}=\frac{\delta P_{\mu}(\lambda)}{\delta P^{\nu}\left(\lambda^{\prime}\right)}=\eta_{\mu \nu} \Delta_{+}\left(\lambda, \lambda^{\prime}\right)
$$

More generally the Poisson bracket for two canonical variables $A(\lambda), B(\lambda)$ has the form

$$
\left\{A(\lambda), B\left(\lambda^{\prime}\right)\right\}=\int_{0}^{\pi} d \bar{\lambda}\left[\frac{\delta A(\lambda)}{\delta P_{\mu}(\bar{\lambda})} \frac{\delta B\left(\lambda^{\prime}\right)}{\delta x^{\mu}(\bar{\lambda})}-\frac{\delta A(\lambda)}{\delta x^{\mu}(\bar{\lambda})} \frac{\delta B\left(\lambda^{\prime}\right)}{\delta P_{\mu}(\bar{\lambda})}\right] .
$$

Since there can be a dependence on $x^{\prime}(\tau, \lambda)$, we must check if some boundary term is present $[21,22]$.

The naive center-of-mass coordinates of the string are

$$
X^{\mu}(\tau)=\frac{1}{2 \pi} \int_{-\pi}^{\pi} d \lambda x^{\mu}(\tau, \lambda), \quad P^{\mu}=\frac{1}{2} \int_{-\pi}^{\pi} d \lambda P^{\mu}(\tau, \lambda)
$$

where $P^{\mu}$ is the conserved generator of the space-time translations. The following relative coordinates have been introduced in Ref.[10]

$$
\begin{aligned}
y^{\mu}(\tau, \lambda) & =-x^{\prime \mu}(\tau, \lambda)=-y^{\mu}(\tau,-\lambda), \\
\mathcal{P}^{\mu}(\tau, \lambda) & =\int_{0}^{\lambda} d \lambda^{\prime} P^{\mu}\left(\tau, \lambda^{\prime}\right)-\frac{\lambda}{\pi} P^{\mu}=-\mathcal{P}^{\mu}(\tau,-\lambda)=\mathcal{P}^{\mu}(\tau, \lambda+2 n \pi),
\end{aligned}
$$

with the following properties

$$
\begin{aligned}
& \int_{-\pi}^{\pi} d \lambda y^{\mu}(\tau, \lambda)=\int_{-\pi}^{\pi} d \lambda \mathcal{P}^{\mu}(\tau, \lambda)=0 \\
& \mathcal{P}^{\mu}(0)=\mathcal{P}^{\mu}( \pm \pi)=0, \quad \rightarrow \int_{-\pi}^{\pi} d \lambda \mathcal{P}^{\prime \mu}(\tau, \lambda)=0
\end{aligned}
$$

and the following inversion 


$$
\begin{aligned}
& x^{\mu}(\tau, \lambda)=X^{\mu}(\tau)+\frac{1}{2 \pi} \int_{-\pi}^{\pi} d \lambda_{1} \int_{0}^{\lambda_{1}} d \lambda_{2} y^{\mu}\left(\tau, \lambda_{2}\right)-\int_{0}^{\lambda} d \lambda_{2} y^{\mu}\left(\tau, \lambda_{2}\right), \\
& P^{\mu}(\tau, \lambda)=\frac{1}{\pi} P^{\mu}+\mathcal{P}^{\prime \mu}(\tau, \lambda) .
\end{aligned}
$$

It may be checked that the coordinates (2.24) and (2.25) constitute a basis of canonical variables

$$
\left\{X^{\mu}, P^{\nu}\right\}=-\eta^{\mu \nu}, \quad\left\{y^{\mu}(\tau, \lambda), \mathcal{P}^{\nu}\left(\tau, \lambda^{\prime}\right)\right\}=-\eta^{\mu \nu} \Delta_{-}\left(\lambda, \lambda^{\prime}\right)
$$

with all the other Poisson brackets vanishing. Here $\Delta_{-}\left(\lambda, \lambda^{\prime}\right)$ is the odd delta function with period $2 \pi$ defined in Eq.(A2).

The constraints implied by equation (2.6) are

$$
\begin{aligned}
& \bar{\chi}_{1}(\tau, \lambda)=\bar{\chi}_{1}(\tau,-\lambda)=P^{2}(\tau, \lambda)+N^{2} x^{\prime 2}(\tau, \lambda) \approx 0, \\
& \bar{\chi}_{2}(\tau, \lambda)=-\bar{\chi}_{2}(\tau,-\lambda)=P(\tau, \lambda) \cdot x^{\prime}(\tau, \lambda) \approx 0 .
\end{aligned}
$$

The following equivalent expression is given in terms of the functions $A_{ \pm}^{\mu}(\tau, \lambda)=$

$$
\begin{gathered}
A_{\mp}^{\mu}(\tau,-\lambda)=P^{\mu}(\tau, \lambda) \pm N x^{\prime \mu}(\tau, \lambda)=\frac{1}{\pi} P^{\mu}+\mathcal{P}^{\prime \mu}(\tau, \lambda) \mp N y^{\mu}(\tau, \lambda)=\frac{\partial}{\partial \lambda} B_{ \pm}^{\mu}(\tau, \lambda)^{5} \\
\bar{\chi}_{ \pm}(\tau, \lambda)=\bar{\chi}_{\mp}(\tau,-\lambda)=A^{2}{ }_{ \pm}(\tau, \lambda) \approx 0, \quad \bar{\chi}_{1}=\frac{1}{2}\left(\bar{\chi}_{+}+\bar{\chi}_{-}\right), \quad \bar{\chi}_{2}=\frac{1}{4 N}\left(\bar{\chi}_{+}-\bar{\chi}_{-}\right) .
\end{gathered}
$$

They satisfy the algebra [10]

$$
\begin{aligned}
& \left\{\bar{\chi}_{ \pm}\left(\tau, \lambda_{1}\right), \bar{\chi}_{ \pm}\left(\tau, \lambda_{2}\right)\right\}=\mp 2 N\left(\bar{\chi}_{ \pm}\left(\tau, \lambda_{1}\right)+\bar{\chi}_{ \pm}\left(\tau, \lambda_{2}\right)\right)\left(\Delta^{\prime}{ }_{+}\left(\lambda_{1}, \lambda_{2}\right)+\Delta^{\prime}{ }_{-}\left(\lambda_{1}, \lambda_{2}\right)\right), \\
& \left\{\bar{\chi}_{+}\left(\tau, \lambda_{1}\right), \bar{\chi}_{-}\left(\tau, \lambda_{2}\right)\right\}=-2 N\left(\bar{\chi}_{+}\left(\tau, \lambda_{1}\right)+\bar{\chi}_{-}\left(\tau, \lambda_{2}\right)\right)\left(\Delta^{\prime}{ }_{+}\left(\lambda_{1}, \lambda_{2}\right)-\Delta^{\prime}{ }_{-}\left(\lambda_{1}, \lambda_{2}\right)\right), \\
& \left\{\bar{\chi}_{2}\left(\tau, \lambda_{1}\right), \bar{\chi}_{2}\left(\tau, \lambda_{2}\right)\right\}=-\left(\bar{\chi}_{2}\left(\tau, \lambda_{2}\right) \Delta_{+}^{\prime}\left(\lambda_{1}, \lambda_{2}\right)+\bar{\chi}_{2}\left(\tau, \lambda_{1}\right) \Delta^{\prime}\left(\lambda_{1}, \lambda_{2}\right)\right) .
\end{aligned}
$$

Therefore the constraints are $1^{\text {st }}$-class, but they are in weak involution; the algebra (2.31) is the universal Dirac algebra of reparametrization.

\footnotetext{
${ }^{5}$ As shown in Ref.[10] one has $x^{\mu}(\tau, \lambda)=\frac{1}{2 N}\left(B_{+}^{\mu}(\tau, \lambda)-B_{-}^{\mu}(\tau, \lambda)\right)$ and $P^{\mu}(\tau, \vec{\lambda})=\frac{1}{2}\left(A_{+}^{\mu}(\tau, \lambda)+\right.$ $\left.A_{-}^{\mu}(\tau, \lambda)\right)$ with $B_{ \pm}^{\mu}(\tau, \lambda)=-B_{\mp}^{\mu}(\tau,-\lambda)=\frac{\lambda}{\pi} P^{\mu}+\mathcal{P}^{\mu}(\tau, \lambda) \pm N x^{\mu}(\tau, \lambda), B_{ \pm}^{\mu}(\tau, \lambda+2 n \pi)=$ $B_{ \pm}^{\mu}(\tau, \lambda)+2 n P^{\mu}, B_{ \pm}^{\mu}(\tau, \pi)-B_{ \pm}^{\mu}(\tau,-\pi)=\int_{-\pi}^{\pi} d \lambda A_{ \pm}^{\mu}(\tau, \lambda)=2 P^{\mu}$. The Poisson brackets of the functions $A_{ \pm}^{\mu}$ are $\left\{A_{ \pm}^{\mu}\left(\tau, \lambda_{1}\right), A_{ \pm}^{\nu}\left(\tau, \lambda_{2}\right)\right\}=\mp N \eta^{\mu \nu}\left(\Delta^{\prime}{ }_{+}\left(\lambda_{1}, \lambda_{2}\right)+\Delta^{\prime}{ }_{-}\left(\lambda_{1}, \lambda_{2}\right)\right),\left\{A_{ \pm}^{\mu}\left(\tau, \lambda_{1}\right), A_{\mp}^{\nu}\left(\tau, \lambda_{2}\right)\right\}=$ $\mp N \eta^{\mu \nu}\left(\Delta^{\prime}{ }_{+}\left(\lambda_{1}, \lambda_{2}\right)-\Delta^{\prime}{ }_{-}\left(\lambda_{1}, \lambda_{2}\right)\right)$.
} 
The Poincare' generators are $\left[Y^{\mu}(\tau, \lambda)=x^{\mu}(\tau, \lambda)-X^{\mu}(\tau)\right]$

$$
\begin{aligned}
P^{\mu} & =\frac{1}{2} \int_{-\pi}^{\pi} d \lambda P^{\mu}(\tau, \lambda) \\
J^{\mu \nu} & =\frac{1}{2} \int_{-\pi}^{\pi} d \lambda\left[x^{\mu}(\tau, \lambda) P^{\nu}(\tau, \vec{\lambda})-x^{\nu}(\tau, \lambda) P^{\mu}(\tau, \lambda)\right]=L^{\mu \nu}+S^{\mu \nu}= \\
& =X^{\mu}(\tau) P^{\nu}-X^{\nu}(\tau) P^{\mu}+\frac{1}{2} \int_{-\pi}^{\pi} d \lambda\left[Y^{\mu}(\tau, \lambda) \mathcal{P}^{\nu}(\tau, \lambda)-Y^{\nu}(\tau, \lambda) \mathcal{P}^{\mu}(\tau, \lambda)\right]
\end{aligned}
$$

After this review of the standard descriptions of the open Nambu string, we will reformulate it as a parametrized Minkowski theory in the next Section. We will define different configuration variables, but we will save the periodicity conditions and the Poisson structure with the $\Delta_{ \pm}$delta functions. 


\section{THE NAMBU STRING AS A PARAMETRIZED MINKOWSKI THEORY.}

As was done for scalar relativistic particles in Refs.[1, 3-6], we can also describe the open Nambu string by means of a parametrized Minkowski theory.

To formulate this theory we need the $3+1$ point of view, in which we assign: a) the world-line of an arbitrary time-like observer; b) an admissible $3+1$ splitting of Minkowski space-time, namely a nice foliation with space-like instantaneous 3-spaces (i.e. a clock synchronization convention). This allows one to define a global non-inertial frame centered on the observer and to use observer-dependent Lorentz-scalar radar 4-coordinates $\sigma^{A}=$ $\left(\tau ; \sigma^{r}\right)$, where $\tau$ is a monotonically increasing function of the proper time of the observer and $\sigma^{r}$ are curvilinear 3-coordinates on the instantaneous 3-spaces $\Sigma_{\tau}$ having the observer as origin. If $x^{\mu} \mapsto \sigma^{A}(x)$ is the coordinate transformation from the inertial Cartesian 4coordinates $x^{\mu}$ to radar coordinates, its inverse $\sigma^{A} \mapsto x^{\mu}=z^{\mu}\left(\tau, \sigma^{r}\right)$ defines the embedding functions $z^{\mu}\left(\tau, \sigma^{r}\right)$ describing the 3-spaces $\Sigma_{\tau}$ as embedded 3-manifold into Minkowski spacetime. The induced 4-metric on $\Sigma_{\tau}$ is the following functional of the embedding ${ }^{4} g_{A B}\left(\tau, \sigma^{r}\right)=$ $\left[z_{A}^{\mu} \eta_{\mu \nu} z_{B}^{\nu}\right]\left(\tau, \sigma^{r}\right)$, where $z_{A}^{\mu}=\partial z^{\mu} / \partial \sigma^{A}$. While the 4 -vectors $z_{r}^{\mu}\left(\tau, \sigma^{u}\right)$ are tangent to $\Sigma_{\tau}$, so that the unit normal $l^{\mu}\left(\tau, \sigma^{u}\right)$ is proportional to $\epsilon_{\alpha \beta \gamma}^{\mu}\left[z_{1}^{\alpha} z_{2}^{\beta} z_{3}^{\gamma}\right]\left(\tau, \sigma^{u}\right)$, we have $z_{\tau}^{\mu}\left(\tau, \sigma^{r}\right)=$ $\left[N l^{\mu}+N^{r} z_{r}^{\mu}\right]\left(\tau, \sigma^{r}\right)\left(N\left(\tau, \sigma^{r}\right)=\epsilon\left[z_{\tau}^{\mu} l_{\mu}\right]\left(\tau, \sigma^{r}\right)\right.$ and $N_{r}\left(\tau, \sigma^{r}\right)=-\epsilon g_{\tau r}\left(\tau, \sigma^{r}\right)$ are the lapse and shift functions).

The foliation is nice and admissible if it satisfies the conditions:

1) $N\left(\tau, \sigma^{r}\right)>0$ in every point of $\Sigma_{\tau}$ (the 3 -spaces never intersect, avoiding the coordinate singularity of Fermi coordinates);

2) ${ }^{4} g_{\tau \tau}\left(\tau, \sigma^{r}\right)>0$, so as to avoid the coordinate singularity of the rotating disk, and with the positive-definite 3 -metric ${ }^{3} g_{r s}\left(\tau, \sigma^{u}\right)=-{ }^{4} g_{r s}\left(\tau, \sigma^{u}\right)$ having three positive eigenvalues (these are the Møller conditions [3]);

3) all the 3-spaces $\Sigma_{\tau}$ must tend to the same space-like hyper-plane at spatial infinity (so that there are always asymptotic inertial observers to be identified with the fixed stars).

In parametrized Minkowski theories one considers any isolated system (particles, strings, fields, fluids) admitting a Lagrangian description, because it allows, through the coupling to an external gravitational field, the determination of the matter energy-momentum tensor and of the ten conserved Poincare' generators $P^{\mu}$ and $J^{\mu \nu}$ (assumed finite) of every configuration of the system. Then one replaces the external gravitational 4-metric in the coupled Lagrangian with the 4-metric $g_{A B}\left(\tau, \sigma^{r}\right)$ of an admissible $3+1$ splitting of Minkowski space-time and the matter fields with new ones knowing the instantaneous 3 -spaces $\Sigma_{\tau}$. For instance for a relativistic particle with world-line $x^{\mu}(\tau)$ we must make a choice of its energy sign: then it will be described by 3-coordinates $\eta^{r}(\tau)$ defined by the intersection of the world-line with $\Sigma_{\tau}: x^{\mu}(\tau)=z^{\mu}\left(\tau, \eta^{r}(\tau)\right)$. Differently from all the previous approaches to relativistic mechanics, the dynamical configuration variables are the 3 -coordinates $\eta_{i}^{r}(\tau)$ and not the world-lines $x_{i}^{\mu}(\tau)$ (to rebuild them in an arbitrary frame we need the embedding defining that frame!).

With this procedure we get a Lagrangian depending on the given matter and on the embedding $z^{\mu}\left(\tau, \sigma^{r}\right)$, which is invariant under frame-preserving diffeomorphisms. As a consequence, there are four first-class constraints (an analogue of the super-Hamiltonian and super-momentum constraints of canonical gravity) implying that the embeddings $z^{\mu}\left(\tau, \sigma^{r}\right)$ are gauge variables, so that all the admissible non-inertial or inertial frames are gauge equivalent, namely physics does not depend on the clock synchronization convention and on the 
choice of the 3-coordinates $\sigma^{r}$.

Let us now consider the Nambu action re-written on space-like hyper-surfaces $\Sigma_{\tau}$, leaves of an admissible $3+1$ splitting of Minkowski space-time, identified by the embeddings $x^{\mu}=$ $z^{\mu}(\tau, \vec{\sigma})$. Let the world-sheet of the string in Minkowski space-time be parametrized as

$$
\begin{aligned}
x^{\mu}(\tau, \lambda)= & z^{\mu}\left(\tau, \eta^{r}(\tau, \lambda)\right), \\
& x^{\prime \mu}(\tau, \lambda)=z_{r}^{\mu}\left(\tau, \eta^{u}(\tau, \lambda)\right) \eta^{\prime r}(\tau, \lambda), \\
& \dot{x}^{\mu}(\tau, \lambda)=z_{\tau}^{\mu}\left(\tau, \eta^{u}(\tau, \lambda)\right)+z_{r}^{\mu}\left(\tau, \eta^{u}(\tau, \lambda)\right) \dot{\eta}^{r}(\tau, \lambda), \\
& \dot{x}^{2}(\tau, \lambda)={ }^{4} g_{\tau \tau}\left(\tau, \eta^{u}(\tau, \lambda)\right)+2{ }^{4} g_{\tau r}\left(\tau, \eta^{u}(\tau, \lambda)\right) \dot{\eta}^{r}(\tau, \lambda)+ \\
+ & { }^{4} g_{r s}\left(\tau, \eta^{u}(\tau, \lambda)\right) \dot{\eta}^{r}(\tau, \lambda) \dot{\eta}^{s}(\tau, \lambda), \\
& x^{\prime 2}(\tau, \lambda)={ }^{4} g_{r s}\left(\tau, \eta^{u}(\tau, \lambda) \eta^{\prime r}(\tau, \lambda)\right) \eta^{\prime s}(\tau, \lambda), \\
& \left.\dot{x}(\tau, \lambda) \cdot x^{\prime}(\tau, \lambda)={ }^{4} g_{\tau s}\left(\tau, \eta^{u}(\tau, \lambda)\right)+{ }^{4} g_{r s}\left(\tau, \eta^{u}(\tau, \lambda)\right) \cdot \eta^{r}(\tau, \lambda)\right) \eta^{s}(\tau, \lambda) .
\end{aligned}
$$

Namely on each instantaneous 3-space $\Sigma_{\tau}$ the string is described by the curvilinear coordinates $\eta^{r}(\tau, \lambda)$ with $\lambda \in(0, \pi)$ and by conjugate 3-momenta $\kappa_{r}(\tau, \lambda)$. We use the notation $\dot{\eta}^{r}(\tau, \lambda)=\frac{\partial \eta^{r}(\tau, \lambda)}{\partial \tau}$ and $\eta^{\prime}(\tau, \lambda)=\frac{\partial \eta^{r}(\tau, \lambda)}{\partial \lambda}$ for the partial derivatives of $\eta^{r}(\tau, \lambda)$ with respect to $\tau$ and $\lambda$, respectively. We have two different theories for the two signs of the total energy $\operatorname{sign} P^{o}$. We shall consider only the sector with $P^{o}>0$.

Like the world-lines in the case of particles, the coordinates $x^{\mu}(\tau, \lambda)$ are derived quantities and the old 4-momenta $P^{\mu}(\tau, \lambda)$ are not defined. In the case of particles one can define a derived quantity $P^{\mu}$ satisfying the mass-shell constraint $P^{2}=m^{2}$. Also in the string case in the inertial frames one can define the following 4 -vector

$$
\begin{aligned}
P^{\mu}(\tau, \lambda) & = \pm l^{\mu}\left(\tau, \eta^{u}(\tau, \lambda)\right) \sqrt{N^{2}{ }^{4} g_{r s}\left(\tau, \eta^{u}(\tau, \lambda)\right) \eta^{\prime r}(\tau, \lambda) \eta^{\prime s}(\tau, \lambda)-\gamma^{r s}\left(\tau, \eta^{u}(\tau, \lambda)\right) \kappa_{r}(\tau, \lambda) \kappa_{s}(\tau, \lambda)}+ \\
& +\kappa_{r}(\tau, \lambda) \gamma^{r s}\left(\tau, \eta^{u}(\tau, \lambda)\right) z_{s}^{\mu}\left(\tau, \eta^{u}(\tau, \lambda)\right)
\end{aligned}
$$

satisfying $P^{2}(\tau, \lambda)+N^{2} x^{\prime 2}(\tau, \lambda)=0$.

Only the transversality constraints, i.e. the counterpart of $P(\tau, \lambda) \cdot x^{\prime}(\tau, \lambda) \approx 0$, will remain. They are the generators of the passive diffeomorphisms along the string and say that the longitudinal degree of freedom is a gauge variable.

The action (2.1) becomes 


$$
\begin{aligned}
S & =\int d \tau \int_{0}^{\pi} d \lambda L(\tau, \lambda) \\
L(\tau, \lambda) & =-N \int d^{3} \sigma \delta^{3}\left(\sigma^{a}-\eta^{a}(\tau, \lambda)\right)\left[\left(-{ }^{4} g_{\tau \tau}{ }^{4} g_{r s}+{ }^{4} g_{\tau r}{ }^{4} g_{\tau s}\right) \eta^{\prime r}(\tau, \lambda) \eta^{\prime s}(\tau, \lambda)+\right. \\
& +\left({ }^{4} g_{r s}{ }^{4} g_{u v}-{ }^{4} g_{r u}{ }^{4} g_{s v}\right) \dot{\eta}^{r}(\tau, \lambda) \eta^{s}(\tau, \lambda) \dot{\eta}^{u}(\tau, \lambda) \eta^{\prime v}(\tau, \lambda)+ \\
& \left.+2\left({ }^{4} g_{r s}{ }^{4} g_{\tau u}-{ }^{4} g_{u s}{ }^{4} g_{\tau r}\right) \dot{\eta}^{r}(\tau, \lambda) \eta^{\prime s}(\tau, \lambda) \eta^{u}(\tau, \lambda)\right]^{\frac{1}{2}}\left(\tau, \sigma^{u}\right)= \\
& \stackrel{\text { def }}{=}-N \int d^{3} \sigma \delta^{3}\left(\sigma^{a}-\eta^{a}(\tau, \lambda)\right) \sqrt{-G\left(z^{\rho}\left(\tau, \sigma^{u}\right), \eta^{u}(\tau, \lambda)\right)}
\end{aligned}
$$

The canonical momenta conjugate to the configuration variables $z^{\mu}(\tau, \vec{\sigma})$ and $\vec{\eta}(\tau, \lambda)$ are $\left(\mathcal{N}(\tau, \vec{\sigma})=\sqrt{\frac{g}{\gamma}}(\tau, \vec{\sigma}), \mathcal{N}^{r}(\tau, \vec{\sigma})=\gamma^{r s}(\tau, \vec{\sigma}){ }^{4} g_{\tau s}(\tau, \vec{\sigma})\right.$ are the lapse and shift functions respectively; $\gamma^{r s}$ is the inverse of ${ }^{4} g_{r s}$ )

$$
\begin{aligned}
& \rho_{\mu}\left(\tau, \sigma^{u}\right)=-\frac{\delta S}{\delta z_{\tau}^{\mu}\left(\tau, \sigma^{u}\right)}= \\
& =\int_{0}^{\pi} d \lambda \delta^{3}\left(\sigma^{a}-\eta^{a}(\tau, \lambda)\right) \frac{N}{\sqrt{-G\left(z^{\rho}\left(\tau, \sigma^{u}\right), \eta^{u}(\tau, \lambda)\right)}} \\
& \times\left(l_{\mu}\left(\tau, \sigma^{u}\right)\left[-\mathcal{N}^{4} g_{r s} \eta^{\prime r}(\tau, \lambda) \eta^{\prime s}(\tau, \lambda)\right](\tau, \vec{\sigma})+\right. \\
& +z_{s \mu}\left(\tau, \sigma^{u}\right) \gamma^{s r}\left(\tau, \sigma^{u}\right)\left[-{ }^{4} g_{r u}\left(\mathcal{N}^{u}+\dot{\eta}^{u}(\tau, \lambda)\right)^{4} g_{m n} \eta^{\prime m}(\tau, \lambda) \eta^{\prime n}(\tau, \lambda)+\right. \\
& \left.\left.+{ }^{4} g_{r u} \eta^{u}(\tau, \lambda){ }^{4} g_{m n}\left(\mathcal{N}^{m}+\dot{\eta}^{m}(\tau, \lambda)\right) \eta^{\prime n}(\tau, \lambda)\right]\left(\tau, \sigma^{u}\right)\right), \\
& \kappa_{r}(\tau, \lambda)=-\frac{\delta S}{\delta \dot{\eta}^{r}(\tau, \lambda)}= \\
& =\frac{N}{\sqrt{-G\left(z^{\rho}\left(\tau, \eta^{u}(\tau, \lambda)\right), \eta^{u}(\tau, \lambda)\right)}}\left[\left({ }^{4} g_{r s}{ }^{4} g_{u v}-{ }^{4} g_{r u}{ }^{4} g_{s v}\right) \dot{\eta}^{u}(\tau, \lambda)+\right. \\
& \left.+{ }^{4} g_{r s}{ }^{4} g_{\tau v}-{ }^{4} g_{\tau r}{ }^{4} g_{s v}\right]\left(\tau, \eta^{u}(\tau, \lambda)\right) \eta^{s}(\tau, \lambda) \eta^{\prime}{ }^{v}(\tau, \lambda),
\end{aligned}
$$

with $\left\{\eta^{r}(\tau, \lambda), \kappa_{s}\left(\tau, \lambda^{\prime}\right)\right\}=-\delta_{s}^{r} \delta\left(\lambda-\lambda^{\prime}\right)$.

With the extension $\eta^{r}(\tau, \lambda)=\eta^{r}(\tau,-\lambda)=\eta^{r}(\tau, \lambda+2 n \pi), \kappa_{r}(\tau, \lambda)=\kappa_{r}(\tau,-\lambda)=\kappa_{r}(\tau, \lambda+$ $2 n \pi)$, we have $\left\{\eta^{r}(\tau, \lambda), \kappa_{s}\left(\tau, \lambda_{1}\right)\right\}=\delta_{s}^{r} \Delta_{+}\left(\lambda, \lambda_{1}\right)=\delta_{s}^{r} \delta\left(\lambda-\lambda_{1}\right)$ for $\lambda, \lambda_{1} \in(0, \pi)$ with the Poisson structure of Eq. $(2.21)$.

Besides the constraints implying the independence from the foliation

$$
\begin{aligned}
\mathcal{H}_{\mu}\left(\tau, \sigma^{u}\right) & =\rho_{\mu}\left(\tau, \sigma^{u}\right)-\int_{0}^{\pi} d \lambda \delta^{3}\left(\sigma^{a}-\eta^{a}(\tau, \lambda)\right)\left(z_{s \mu}\left(\tau, \sigma^{u}\right) \gamma^{s r}\left(\tau, \sigma^{u}\right) \kappa_{r}(\tau, \lambda)+\right. \\
& \left.+l_{\mu}\left(\tau, \sigma^{u}\right) \sqrt{N^{2}{ }^{4} g_{r s}\left(\tau, \sigma^{u}\right) \eta^{\prime r}(\tau, \lambda) \eta^{\prime} s(\tau, \lambda)-\gamma^{r s}\left(\tau, \sigma^{u}\right) \kappa_{r}(\tau, \lambda) \kappa_{s}(\tau, \lambda)}\right) \approx 0
\end{aligned}
$$


we get the following transversality constraints

$$
\bar{\chi}(\tau, \lambda)=-\kappa_{r}(\tau, \lambda) \eta^{\prime}(\tau, \lambda) \approx 0 .
$$

All the constraints are first class. In particular we have the following Poisson brackets implying the diffeomorphism algebra (the analogue of the last line in Eqs.(2.31))

$$
\begin{aligned}
\left\{\bar{\chi}(\tau, \lambda), \bar{\chi}\left(\tau, \lambda^{\prime}\right)\right\} & =\bar{\chi}(\tau, \lambda) \frac{\partial \delta\left(\lambda-\lambda^{\prime}\right)}{\partial \lambda}-\bar{\chi}\left(\tau, \lambda^{\prime}\right) \frac{\partial \delta\left(\lambda-\lambda^{\prime}\right)}{\partial \lambda^{\prime}}= \\
& =\bar{\chi}(\tau, \lambda) \Delta_{-}^{\prime}\left(\lambda, \lambda^{\prime}\right)+\bar{\chi}\left(\tau, \lambda^{\prime}\right) \Delta_{+}^{\prime}\left(\lambda, \lambda^{\prime}\right) \approx 0 .
\end{aligned}
$$

The Poincare' generators are

$$
\begin{aligned}
P^{\mu} & =\int d 3 \sigma \rho^{\mu}\left(\tau, \sigma^{u}\right) \\
J^{\mu \nu} & =\int d 3 \sigma\left[z^{\mu} \rho^{\nu}-z^{\nu} \rho^{\mu}\right]\left(\tau, \sigma^{u}\right) .
\end{aligned}
$$

The description of the string in non-inertial frames could be done by using Ref.[3]. Instead in the next Section we will study its rest-frame instant form. 


\section{THE REST-FRAME INSTANT FORM OF THE NAMBU STRING.}

If we restrict ourselves to inertial frames, we can define the inertial rest-frame instant form of dynamics for isolated systems [4-6] by choosing the $3+1$ splitting corresponding to the intrinsic inertial rest frame of the isolated system centered on an inertial observer: the instantaneous 3-spaces, named Wigner 3-space due to the fact that the 3-vectors inside them are Wigner spin-1 3-vectors, are orthogonal to the conserved 4-momentum $P^{\mu}$ of the configuration.

As said in the Introduction in the inertial rest frames we can give the final solution to the old problem of the relativistic extension of the Newtonian center of mass of an isolated system. In its rest frame there are only three notions of collective variables, which can be built by using only the Poincare' generators (they are non-local quantities knowing the whole $\Sigma_{\tau}$ ): the canonical non-covariant Newton-Wigner center of mass (or center of spin), the non-canonical covariant Fokker-Pryce center of inertia and the non-canonical non-covariant Møller center of energy. All of them tend to the Newtonian center of mass in the non-relativistic limit. As shown in Refs. [4] these three variables can be expressed as known functions of the rest time $\tau$, of the canonically conjugate Jacobi data (frozen Cauchy data) $\vec{z}=M \vec{x}_{N W}(0)\left(\vec{x}_{N W}(\tau)\right.$ is the standard Newton-Wigner 3-position) and $\vec{h}=\vec{P} / M$ $\left(\left\{z^{i}, h^{j}\right\}=\delta^{i j}\right)$, of the invariant mass $M=\sqrt{P^{2}}$ of the system and of its rest spin $\overrightarrow{\bar{S}}$.

As a consequence, every isolated system (i.e. a closed universe) can be visualized as a decoupled non-covariant collective (non-local) pseudo-particle described by the frozen Jacobi data $\vec{z}, \vec{h}$ carrying a pole-dipole structure, namely the invariant mass and the rest spin of the system, and with an associated external realization of the Poincare' group. The universal breaking of Lorentz covariance is connected to the decoupled non-local collective variable $\vec{z}$. As already said in each Wigner 3-space there is an unfaithful inner realization of the Poincare' algebra, whose generators $M, \vec{k} \approx 0, \vec{S}, \overrightarrow{\mathcal{K}} \approx 0$ are built by using the energymomentum tensor of the isolated system. The three pairs of second class constraints $\vec{k} \approx 0$, $\overrightarrow{\mathcal{K}} \approx 0$ (the inner Lorentz boosts are interaction dependent), eliminate the six degrees of freedom of the inner center of mass and identify the observer origin of the 3-coordinates $\sigma^{r}$ with the covariant non-canonical Fokker-Pryce center of inertia. The invariant mass $M$,

which depends only on the Wigner-covariant relative variables (like the rest spin $\overrightarrow{\vec{S}}$ ), is the effective Hamiltonian inside the Wigner 3-spaces as shown in Ref.[4].

The Wigner hyper-planes are defined by the embeddings 


$$
\begin{aligned}
z_{W}^{\mu}(\tau, \vec{\sigma}) & =Y^{\mu}(\tau)+\epsilon_{r}^{\mu}(P) \sigma^{r}, \\
Y^{\mu}(\tau) & =u^{\mu}(P) \tau, \quad z_{W \tau}^{\mu}(\tau, \vec{\sigma})=u^{\mu}(P), z_{W r}^{\mu}(\tau, \vec{\sigma})=\epsilon_{r}^{\mu}(P), \\
\epsilon_{o}^{\mu}(u(P)) & =u^{\mu}(P)=P^{\mu} / \sqrt{P^{2}}=h^{\mu}=\left(\sqrt{1+\vec{h}^{2}} ; \vec{h}\right)=\epsilon_{o}^{\mu}(\vec{h}), \\
\epsilon_{r}^{\mu}(u(P)) & =\left(-u_{r}(P) ; \delta_{r}^{i}-\frac{u^{i}(P) u_{r}(P)}{1+u^{o}(P)}\right)=\epsilon^{\mu}(\vec{h}), \\
\epsilon_{\mu}^{o}(u(P)) & =\eta^{o B} \eta_{\mu \nu} \epsilon_{B}^{\nu}(u(P))=u_{\mu}(P)=h_{\mu}, \\
\epsilon_{\mu}^{r}(u(P)) & =\eta^{r B} \eta_{\mu \nu} \epsilon_{B}^{\nu}(u(P))=\epsilon_{\mu}^{r}(\vec{h}) .
\end{aligned}
$$

where $Y^{\mu}(\tau)$ is the inertial observer corresponding to the Fokker-Pryce 4-center of inertia of the string. The space-like 4 -vectors $\epsilon_{r}^{\mu}(\vec{h})$ together with the time-like one $\epsilon_{o}^{\mu}(\vec{h})$ are the columns of the standard Wigner boost for time-like Poincare' orbits ${ }^{6}$.

The open string and its end points have the following representation $\left[\dot{\vec{\eta}}_{A}(\tau)=\right.$ $\left.\left.\frac{\partial}{\partial \tau} \vec{\eta}(\tau, \lambda)\right|_{\lambda=A}, A=0, \pi\right]$

$$
\begin{gathered}
x^{\mu}(\tau, \lambda)=z_{W}^{\mu}(\tau, \vec{\eta}(\tau, \lambda))=Y^{\mu}(\tau)+\epsilon_{r}^{\mu}(\vec{h}) \eta^{r}(\tau, \lambda), \\
Y^{\mu}(\tau)=\left(\sqrt{1+\vec{h}^{2}}\left(\tau+\frac{\vec{h} \cdot \vec{z}}{M}\right) ; \frac{\vec{z}}{M}+\left(\tau+\frac{\overrightarrow{\vec{h}} \times \vec{z}}{M}\right) \vec{h}+\frac{\vec{h}}{M\left(1+\sqrt{1+\vec{h}^{2}}\right)}\right)=z_{W}^{\mu}(\tau, \overrightarrow{0}), \\
x_{o}^{\mu}(\tau) \stackrel{\text { def }}{=} x^{\mu}(\tau, 0)=Y^{\mu}(\tau)+\epsilon_{r}^{\mu}(\vec{h}) \eta_{o}^{r}(\tau), \quad \vec{\eta}_{o}(\tau) \stackrel{\text { def }}{=} \vec{\eta}(\tau, 0), \\
x_{\pi}^{\mu}(\tau) \stackrel{\text { def }}{=} x^{\mu}(\tau, \pi)=Y^{\mu}(\tau)+\epsilon_{r}^{\mu}(\vec{h}) \eta_{\pi}^{r}(\tau), \quad \vec{\eta}_{\pi}(\tau) \stackrel{\text { def }}{=} \vec{\eta}(\tau, \pi), \\
\dot{x}_{o}^{2}(\tau)=0, \rightarrow \dot{\vec{\eta}}_{o}^{2}=1, \quad \dot{x}_{\pi}^{2}(\tau)=0, \rightarrow \dot{\vec{\eta}}_{\pi}^{2}=1,
\end{gathered}
$$

where the expression of the Fokker-Pryce center of inertia, as a function of $\tau, \vec{z}, \vec{h}, M=\sqrt{P^{2}}$ and $\vec{S}$, was taken from Ref.[4].

This shows that inside the Wigner 3 -spaces the open string is described by the Wigner spin-1 3-vectors $\vec{\eta}(\tau, \lambda), \vec{\kappa}(\tau, \lambda)^{7}$ as independent canonical variables. To them we must add $P^{\mu}$ of Eq.(3.8) and a canonically conjugate collective variable $\tilde{x}^{\mu}$ and then replace them with the final center-of-mass variables $\vec{z}$ and $\vec{h}$.

${ }^{6}$ It sends the time-like four-vector $P^{\mu}$ to its rest-frame form $\stackrel{\circ}{P}^{\mu}=\eta \sqrt{P^{2}}(1 ; \overrightarrow{0})$, where $\eta=\operatorname{sign} P^{o}$. From now on we restrict ourselves to positive energies, i.e. $\eta=1$. While $\epsilon_{o}^{\mu}(u(P))$ and $\epsilon_{\mu}^{o}(u(P))$ are 4-vectors, $\epsilon_{r}^{\mu}(u(P))$ have more complex transformation properties under Lorentz transformations.

${ }^{7}$ Under Lorentz transformations $\Lambda$ these 3 -vectors rotate with Wigner rotations $[\stackrel{\circ}{P}=M(1 ; \overrightarrow{0})$; $P^{\mu}=L(P, \stackrel{\circ}{P})^{\mu}{ }_{\nu} \stackrel{\circ}{P}^{\nu}=\epsilon_{A=\nu}^{\mu}(u(P)) \stackrel{\circ}{P}$ with $L$ the standard Wigner boost] 
It turns out that the relevant collective variable to be added is the external canonical non-covariant 4-center of mass $\tilde{x}^{\mu}(\tau)$. From Ref.[4] we have the following expressions for $\tilde{x}^{\mu}(\tau)$ in terms of $\tau, \vec{z}, \vec{h}, M=\sqrt{P^{2}}$ and $\vec{S}$, and for the Lorentz generators $J^{\mu \nu}$ of Eq.(3.8) (included the definition of the rest-frame spin $\overrightarrow{\bar{S}}$ )

$$
\begin{aligned}
\tilde{x}^{\mu}(\tau) & =\left(\sqrt{1+\vec{h}^{2}}\left(\tau+\frac{\vec{h} \cdot \vec{z}}{M}\right) ; \frac{\vec{z}}{M}+\left(\tau+\frac{\vec{h} \cdot \vec{z}}{M}\right) \vec{h}\right)= \\
& =z_{W}^{\mu}(\tau, \tilde{\vec{\sigma}})=Y^{\mu}(\tau)+\left(0 ; \frac{-\vec{S} \times \vec{h}}{M\left(1+\sqrt{1+\vec{h}^{2}}\right)}\right), \\
J^{\mu \nu} & =\left[h^{\mu} \epsilon_{r}^{\nu}(\vec{h})-h^{\nu} \epsilon_{r}^{\mu}(\vec{h})\right] \bar{S}^{o r}+\epsilon_{r}^{\mu}(\vec{h}) \epsilon_{s}^{\nu}(\vec{h}) \bar{S}^{r s} \\
\bar{S}^{r s} & \equiv \int_{o}^{\pi} d \lambda\left[\eta^{r} k^{s}-\eta^{s} k^{r}\right](\tau, \lambda), \\
\bar{S}^{o r} & \equiv-\int_{o}^{\pi} d \lambda \eta^{r}(\tau, \lambda) \sqrt{-N^{2}\left(\partial_{\lambda} \vec{\eta}(\tau, \lambda)\right)^{2}+\vec{\kappa}^{2}(\tau, \lambda)}, \\
\tilde{S}^{i j} & =\delta^{i r} \delta^{j s} \bar{S}^{r s}, \quad \tilde{S}^{o i}=-\frac{\delta^{i r} \bar{S}^{r s} P^{s}}{P^{o}+M} .
\end{aligned}
$$

The external Poincare' generators are [while $i, j .$. are Euclidean indices, $r, s .$. are Wigner spin-1 indices; $\tilde{S}^{\mu \nu}$ is given in Eq.(4.3)]

$$
\begin{aligned}
& P^{\mu}, \quad J^{\mu \nu}=x_{s}^{\mu} P^{\nu}-x_{s}^{\nu} P^{\mu}+S^{\mu \nu}=\tilde{x}^{\mu} P^{\nu}-\tilde{x}^{\nu} P^{\mu}+\tilde{S}^{\mu \nu}, \\
& P^{o}=\sqrt{M^{2}+\vec{P}^{2}}=M \sqrt{1+\vec{h}^{2}}, \quad \vec{P}=M \vec{h}, \\
& J^{i j}=\tilde{x}^{i} P^{j}-\tilde{x}^{j} P^{i}+\delta^{i r} \delta^{j s} \epsilon^{r s u} \bar{S}^{u}=z^{i} h^{j}-z^{j} h^{i}+\epsilon^{i j u} \bar{S}^{u}, \\
& K^{i}=J^{o i}=\tilde{x}^{o} P^{i}-\tilde{x}^{i} \sqrt{M^{2}+\vec{P}^{2}}-\frac{\delta^{i r} P^{s} \epsilon^{r s u} \bar{S}^{u}}{M+\sqrt{M^{2}+\vec{P}^{2}}}= \\
& =-\sqrt{1+\vec{h}^{2}} z^{i}+\frac{(\vec{S} \times \vec{h})^{i}}{1+\sqrt{1+\vec{h}^{2}}} .
\end{aligned}
$$

$$
\begin{aligned}
R_{\nu}^{\mu}(\Lambda, P) & =\left[L(\stackrel{\circ}{P}, P) \Lambda^{-1} L(\Lambda P, \stackrel{\circ}{P})\right]_{\nu}^{\mu}=\left(\begin{array}{cc}
1 & 0 \\
0 & R_{j}^{i}(\Lambda, P)
\end{array}\right), \\
R_{j}^{i}(\Lambda, P) & =\left(\Lambda^{-1}\right)_{j}^{i}-\frac{\left(\Lambda^{-1}\right)^{i}{ }_{o} P_{\beta}\left(\Lambda^{-1}\right)^{\beta}{ }_{j}}{P_{\rho}\left(\Lambda^{-1}\right)_{o}+\sqrt{P^{2}}}- \\
& -\frac{P^{i}}{P^{o}+\sqrt{P^{2}}}\left[\left(\Lambda^{-1}\right)^{o}{ }_{j}-\frac{\left(\left(\Lambda^{-1}\right)_{o}^{o}-1\right) P_{\beta}\left(\Lambda^{-1}\right)^{\beta} j}{P_{\rho}\left(\Lambda^{-1}\right)_{o}+\sqrt{P^{2}}}\right] .
\end{aligned}
$$

As a consequence the scalar product of two of these 3 -vectors is a Lorentz scalar. 
Note that both $\tilde{L}^{\mu \nu}=\tilde{x}^{\mu} P^{\nu}-\tilde{x}^{\nu} P^{\mu}$ and $\tilde{S}^{\mu \nu}$ are conserved.

Inside the Wigner 3-spaces the Poincare' generators (3.8) take the following form

$$
\begin{aligned}
P^{\mu} & \approx h^{\mu} \int_{o}^{\pi} d \lambda \sqrt{-N^{2} \vec{\eta}^{\prime 2}(\tau, \lambda)+\vec{\kappa}^{2}(\tau, \lambda)}+N \epsilon_{r}^{\mu}(\vec{h}) \int_{o}^{\pi} d \lambda \kappa^{r}(\tau, \lambda) \approx \\
& \approx h^{\mu} \int_{o}^{\pi} d \lambda \sqrt{-N^{2} \vec{\eta}^{\prime 2}(\tau, \lambda)+\vec{\kappa}^{2}(\tau, \lambda)}, \\
J^{\mu \nu} & \approx\left[h^{\mu} \epsilon_{r}^{\nu}(\vec{h})-h^{\nu} \epsilon_{r}^{\mu}(\vec{h})\right]\left[\tau \int_{o}^{\pi} d \lambda \kappa^{r}(\tau, \lambda)-\right. \\
& \left.-\int_{o}^{\pi} d \lambda \eta^{r}(\tau, \lambda) \sqrt{-N^{2} \vec{\eta}^{\prime 2}(\tau, \lambda)+\vec{\kappa}^{2}(\tau, \lambda)}\right]+ \\
& +\left[\epsilon_{r}^{\mu}(\vec{h}) \epsilon_{s}^{\nu}(\vec{h})-\epsilon_{r}^{\nu}(\vec{h}) \epsilon_{s}^{\mu}(\vec{h})\right] \int_{o}^{\pi} d \lambda \eta^{r}(\tau, \lambda) \kappa^{s}(\tau, \lambda) \approx \\
& \approx\left[h^{\mu} \epsilon_{r}^{\nu}(\vec{h})-h^{\nu} \epsilon_{r}^{\mu}(\vec{h})\right]\left[-\int_{o}^{\pi} d \lambda \eta^{r}(\tau, \lambda) \sqrt{-N^{2} \vec{\eta}^{\prime 2}(\tau, \lambda)+\vec{\kappa}^{2}(\tau, \lambda)}\right]+ \\
& +\left[\epsilon_{r}^{\mu}(\vec{h}) \epsilon_{s}^{\nu}(\vec{h})-\epsilon_{r}^{\nu}(\vec{h}) \epsilon_{s}^{\mu}(\vec{h})\right] \int_{o}^{\pi} d \lambda \eta^{r}(\tau, \lambda) \kappa^{s}(\tau, \lambda),
\end{aligned}
$$

As a consequence, inside the Wigner hyper-plane there is the following unfaithful realization of the Poincare' algebra, whose inner generators are

$$
\begin{aligned}
M & =\int_{0}^{\pi} d \lambda \sqrt{N^{2}\left(\partial_{\lambda} \vec{\eta}(\tau, \lambda)\right)^{2}+\vec{\kappa}^{2}(\tau, \lambda)}, \\
\vec{k} & =\int_{-\pi}^{\pi} d \lambda \vec{\kappa}(\tau, \lambda) \approx 0, \\
\overrightarrow{\mathcal{J}} & =\vec{S}=\int_{o}^{\pi} d \lambda \vec{\eta}(\tau, \lambda) \times \vec{\kappa}(\tau, \lambda), \\
\mathcal{K}^{r} & =\bar{S}^{\text {or }}=-\int_{o}^{\pi} d \lambda \eta^{r}(\tau, \lambda) \sqrt{N^{2}\left(\partial_{\lambda} \vec{\eta}(\tau, \lambda)\right)^{2}+\vec{\kappa}^{2}(\tau, \lambda)} \approx 0 .
\end{aligned}
$$

As already said, while $\vec{k} \approx 0$ is the rest-frame condition, $\mathcal{K}^{r} \approx 0$ eliminates the inner center of mass inside the Wigner hyper-planes and, as shown in Ref.[4], implies that the embedding (4.1), defining them, is centered on the inertial covariant non-canonical Fokker-Pryce center of inertia $Y^{\mu}(\tau)$. The rest-frame condition $\vec{k} \approx 0$ implies $\dot{x}_{s}^{\mu}(\tau)=\dot{\tilde{x}}^{\mu}(\tau)=\dot{Y}^{\mu}(\tau)=u^{\mu}(P)$, i.e. the velocities are all parallel to $P^{\mu}$, so that there is no classical zitterbewegung.

We must now find a canonical basis of relative variables inside the Wigner 3 -spaces by defining a rest-frame instant form analogue of Eqs. (2.25)-(2.27). This can be done with the following canonical transformation

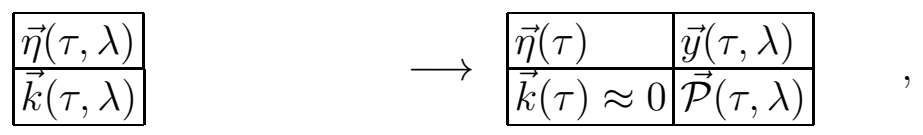




$$
\begin{aligned}
& \vec{\eta}(\tau)=\frac{1}{2 \pi} \int_{-\pi}^{\pi} d \lambda \vec{\eta}(\tau, \lambda), \quad \vec{k}=\frac{1}{2} \int_{-\pi}^{\pi} d \lambda \vec{\kappa}(\tau, \lambda) \approx 0, \\
& \vec{y}(\tau, \lambda)=-\partial_{\lambda} \vec{\eta}(\tau, \lambda), \quad \overrightarrow{\mathcal{P}}(\tau, \lambda)=\int_{0}^{\lambda} d \lambda_{1} \vec{\kappa}\left(\tau, \lambda_{1}\right)-\frac{\lambda}{\pi} \vec{k}, \\
& \int_{-\pi}^{\pi} d \lambda \vec{y}(\tau, \lambda)=\int_{-\pi}^{\pi} d \lambda \overrightarrow{\mathcal{P}}(\tau, \lambda)=0, \\
& \overrightarrow{\mathcal{P}}(\tau, 0)=\overrightarrow{\mathcal{P}}(\tau, \pm \pi)=0, \Rightarrow \int_{-\pi}^{\pi} d \lambda \partial_{\lambda} \overrightarrow{\mathcal{P}}(\tau, \lambda)=0, \\
& \left\{\eta^{i}, k^{j}\right\}=\delta^{i j}, \quad\left\{y^{i}(\tau, \lambda), \mathcal{P}^{j}\left(\tau, \lambda_{1}\right)\right\}=\delta^{i j} \triangle_{-}\left(\lambda, \lambda_{1}\right), \\
& \vec{\eta}(\tau, \lambda)=\vec{\eta}(\tau)+\frac{1}{2 \pi} \int_{-\pi}^{\pi} d \lambda_{1} \int_{\lambda}^{\lambda_{1}} d \lambda_{2} \vec{y}\left(\tau, \lambda_{2}\right) \\
& =\vec{\eta}(\tau)+\vec{\zeta}(\tau, \lambda) \\
& \vec{\kappa}(\tau, \lambda)=\frac{\vec{k}}{\pi}+\partial_{\lambda} \overrightarrow{\mathcal{P}}(\tau, \lambda) \approx \partial_{\lambda} \overrightarrow{\mathcal{P}}(\tau, \lambda), \\
& \vec{\eta}_{o}(\tau)=\vec{\eta}(\tau, 0)=\vec{\eta}(\tau)+\frac{1}{2 \pi} \int_{-\pi}^{\pi} d \lambda_{1} \int_{o}^{\lambda_{1}} d \lambda_{2} \vec{y}\left(\tau, \lambda_{2}\right), \\
& \vec{\eta}_{\pi}(\tau)=\vec{\eta}(\tau, \pi)=\vec{\eta}_{o}(\tau)-\int_{o}^{\pi} d \lambda_{2} \vec{y}\left(\tau, \lambda_{2}\right), \quad \dot{\vec{\eta}}_{o}^{2}(\tau)=\dot{\vec{\eta}}_{\pi}^{2}(\tau)=1, \\
& \vec{S} \approx \int_{-\pi}^{\pi} d \lambda \vec{\zeta}(\tau, \lambda) \times \partial_{\lambda} \overrightarrow{\mathcal{P}}(\tau, \lambda)=\int_{0}^{\pi} d \lambda \vec{y}(\tau, \lambda) \times \overrightarrow{\mathcal{P}}(\tau, \lambda) .
\end{aligned}
$$

In the new canonical basis the inner Poincare' generators and the first class constraints take the following form [in accord with Eq.(3.7)] 


$$
\begin{aligned}
M & \approx \int_{o}^{\pi} d \lambda \sqrt{-N^{2} \vec{y}^{2}(\tau, \lambda)+\left(\partial_{\lambda} \overrightarrow{\mathcal{P}}(\tau, \lambda)\right)^{2}} \\
\mathcal{K}^{i} & \approx-\eta^{i}(\tau) \int_{o}^{\pi} d \lambda \sqrt{-N^{2} \vec{y}^{2}(\tau, \lambda)+\left(\partial_{\lambda} \overrightarrow{\mathcal{P}}(\tau, \lambda)\right)^{2}}- \\
& -\frac{1}{2 \pi} \int_{o}^{\pi} d \lambda \int_{-\pi}^{\pi} d \lambda_{1} \int_{\lambda}^{\lambda_{1}} d \lambda_{2} y^{i}\left(\tau, \lambda_{2}\right) \sqrt{-N^{2} \vec{y}^{2}(\tau, \lambda)+\left(\partial_{\lambda} \overrightarrow{\mathcal{P}}(\tau, \lambda)\right)^{2}} \approx 0, \\
\vec{k} & \approx 0, \\
\vec{S} & \approx \int_{o}^{\pi} d \lambda \vec{y}(\tau, \lambda) \times \overrightarrow{\mathcal{P}}(\tau, \lambda), \\
\chi(\tau, \lambda) & =\vec{\kappa}(\tau, \lambda) \cdot \partial_{\lambda} \vec{\eta}(\tau, \lambda) \approx-\partial_{\lambda} \overrightarrow{\mathcal{P}}(\tau, \lambda) \cdot \vec{y}(\tau, \lambda) \approx 0, \\
\left\{\chi(\tau, \lambda), \chi\left(\tau, \lambda_{1}\right)\right\} & =+\chi(\tau, \lambda) \partial_{\lambda} \delta\left(\lambda-\lambda_{1}\right)-\chi\left(\tau, \lambda_{1}\right) \partial_{\lambda_{1}} \delta\left(\lambda-\lambda_{1}\right)= \\
& =+\chi(\tau, \lambda) \frac{\partial \Delta_{-}\left(\lambda, \lambda_{1}\right)}{\partial \lambda}-\chi\left(\tau, \lambda_{1}\right) \frac{\partial \Delta_{+}\left(\lambda, \lambda_{1}\right)}{\partial \lambda_{1}} .
\end{aligned}
$$

The conditions $\mathcal{K}^{r} \approx 0$ eliminating the inner center of mass give the following determination of the collective variable $\vec{\eta}(\tau)$

$$
\begin{aligned}
\vec{\eta}(\tau) \approx & +\frac{1}{2 \pi \int_{o}^{\pi} d \lambda \sqrt{-N^{2} \vec{y}^{2}(\tau, \lambda)+\left(\partial_{\lambda} \overrightarrow{\mathcal{P}}(\tau, \lambda)\right)^{2}}} \\
& \int_{o}^{\pi} d \lambda \int_{-\pi}^{\pi} d \lambda_{1} \int_{\lambda}^{\lambda_{1}} \vec{y}\left(\tau, \lambda_{2}\right) \sqrt{-N^{2} \vec{y}^{2}(\tau, \lambda)+\left(\partial_{\lambda} \overrightarrow{\mathcal{P}}(\tau, \lambda)\right)^{2}}, \\
\Downarrow & \\
\vec{\eta}(\tau, \lambda) \approx & \frac{1}{2 \pi} \int_{-\pi}^{\pi} d \lambda_{1} \int_{\lambda}^{\lambda_{1}} d \lambda_{2} \vec{y}\left(\tau, \lambda_{2}\right)+\frac{1}{2 \pi \int_{o}^{\pi} d \lambda \sqrt{-N^{2} \vec{y}(\tau, \lambda)+\left(\partial_{\lambda} \overrightarrow{\mathcal{P}}(\tau, \lambda)\right)^{2}}} \\
& \int_{o}^{\pi} d \lambda \int_{-\pi}^{\pi} d \lambda_{1} \int_{\lambda}^{\lambda_{1}} \vec{y}\left(\tau, \lambda_{2}\right) \sqrt{-N^{2} \vec{y}^{2}(\tau, \lambda)+\left(\partial_{\lambda} \overrightarrow{\mathcal{P}}(\tau, \lambda)\right)^{2}}, \\
x^{\mu}(\tau, \lambda)= & Y^{\mu}(\tau)+\epsilon_{r}^{\mu}(\vec{h}) \eta^{r}(\tau, \lambda) .
\end{aligned}
$$

Since we have $\{\vec{k}, \chi(\tau, \lambda)\}=0$, the gauge fixings $\overrightarrow{\mathcal{K}} \approx 0$ lead to the Dirac bracket $\left\{\chi(\tau, \lambda), \chi\left(\tau, \lambda^{\prime}\right)\right\}^{*}=\left\{\chi(\tau, \lambda), \chi\left(\tau, \lambda^{\prime}\right)\right\}$, namely Eqs.(4.8) remain unchanged. 


\section{THE FRENET-SERRET CANONICAL BASIS}

The next step is to find a canonical transformation from the canonical basis (4.7) for the relative variables to a new basis also adapted to the transversality constraints given in Eqs.(4.8). This can be done by using a Frenet-Serret (FS) description of the vectors tangent and normal to the string (see Ref.[23]).

The unit tangent $\hat{t}(\tau, \lambda), \hat{t}^{2}(\tau, \lambda)=1$, to the string inside the Wigner hyper-plane and the final expression of the transversality constraint and of the invariant mass are

$$
\begin{aligned}
& \hat{t}(\tau, \lambda)=-\frac{\vec{y}(\tau, \lambda)}{h(\tau, \lambda)}=\frac{\partial_{\lambda} \vec{\eta}(\tau, \lambda)}{h(\tau, \lambda)} \\
& \Rightarrow \quad h(\tau, \lambda)=\sqrt{\vec{y}^{2}(\tau, \lambda)} \stackrel{\text { def }}{=} \partial_{\lambda} s(\tau, \lambda), \quad\left\{s(\tau, \lambda), s\left(\tau, \lambda_{1}\right)\right\}=0 \\
& \Rightarrow \quad \chi(\tau, \lambda)=h(\tau, \lambda) \partial_{\lambda} \overrightarrow{\mathcal{P}}(\tau, \lambda) \cdot \hat{t}(\tau, \lambda) \approx 0 \\
& \text { or } \quad \pi_{\chi}(\tau, \lambda) \stackrel{\text { def }}{=}+\frac{\chi(\tau, \lambda)}{h(\tau, \lambda)}=\left[\partial_{\lambda} \overrightarrow{\mathcal{P}} \cdot \hat{t}\right](\tau, \lambda) \approx 0 \\
& M=\int_{o}^{\pi} d \lambda \sqrt{-N^{2}\left(\partial_{\lambda} s(\tau, \lambda)\right)^{2}+\left(\partial_{\lambda} \overrightarrow{\mathcal{P}}(\tau, \vec{\lambda})\right)^{2}}
\end{aligned}
$$

The density of invariant mass depends upon the gauge variable $s(\tau, \lambda)$, which describes the arc-length along the string ${ }^{8}$.

In the gauge $s(\tau, \lambda)=\lambda, h(\tau, \lambda)=1, \vec{y}(\tau, \lambda)=-\hat{t}(\tau, \lambda)$ we have

$$
M=\int_{o}^{\pi} d \lambda \sqrt{-N^{2}+\left(\partial_{\lambda} \overrightarrow{\mathcal{P}}(\tau, \vec{\lambda})\right)^{2}}:
$$

let us call this gauge the FS gauge.

Since we have

$$
\begin{aligned}
\left\{y^{r}(\tau, \lambda), \chi\left(\tau, \lambda_{1}\right)\right\} & =\partial_{\lambda_{1}} \eta^{r}\left(\tau, \lambda_{1}\right) \partial_{\lambda} \delta\left(\lambda-\lambda_{1}\right)=+\partial_{\lambda}\left[y^{r}(\tau, \lambda) \delta\left(\lambda-\lambda_{1}\right)\right] \\
& =\partial_{\lambda_{1}} \eta^{r}\left(\tau, \lambda_{1}\right) \partial_{\lambda} \Delta_{+}\left(\lambda, \lambda_{1}\right)=+\partial_{\lambda}\left[y^{r}(\tau, \lambda) \Delta_{-}\left(\lambda, \lambda_{1}\right)\right] \\
\left\{h(\tau, \lambda), \chi\left(\tau, \lambda_{1}\right)\right\} & =+\partial_{\lambda}\left[h(\tau, \lambda) \delta\left(\lambda-\lambda_{1}\right)\right]=+\partial_{\lambda}\left[h(\tau, \lambda) \Delta_{-}\left(\lambda, \lambda_{1}\right)\right] \\
\left\{s(\tau, \lambda), \chi\left(\tau, \lambda_{1}\right)\right\} & =+h(\tau, \lambda) \delta\left(\lambda-\lambda_{1}\right)=+\partial_{\lambda} s(\tau, \lambda) \delta\left(\lambda-\lambda_{1}\right)= \\
& =+h(\tau, \lambda) \Delta_{-}\left(\lambda, \lambda_{1}\right)=+\partial_{\lambda} s(\tau, \lambda) \Delta_{-}\left(\lambda, \lambda_{1}\right)
\end{aligned}
$$

\footnotetext{
${ }^{8}$ If we invert $s=s(\tau, \lambda)$ to $\lambda=\lambda(\tau, s)$, we get $\hat{t}(\tau, \lambda(\tau, s))=\frac{\partial_{\lambda} \vec{\eta}(\tau, \lambda)}{\partial_{\lambda} s(\tau, \lambda)}=\frac{\partial \vec{\eta}(\tau, \lambda(\tau, s))}{\partial s}$.
} 
we get an Abelianization of the transversality constraints with the new Poisson brackets

$$
\begin{gathered}
\left\{s(\tau, \lambda), \pi_{\chi}\left(\tau, \lambda_{1}\right)\right\}=\delta\left(\lambda-\lambda_{1}\right)=\Delta_{-}\left(\lambda, \lambda_{1}\right), \\
\left\{\pi_{\chi}(\tau, \lambda), \pi_{\chi}\left(\tau, \lambda_{1}\right)\right\}=0, \quad\left\{s(\tau, \lambda), s\left(\tau, \lambda_{1}\right)\right\}=0 .
\end{gathered}
$$

On the Euclidean Wigner 3-space $\Sigma_{\tau}$ the open string is described by the Wigner 3-vector $\vec{\eta}(\tau, \lambda)$ with $\lambda \in(0, \pi)$. Let $\hat{t}(\tau, \lambda), \hat{n}(\tau, \lambda)$ and $\hat{b}(\tau, \lambda)$ be the unit tangent, unit normal and unit binormal 3-vectors to the string $\vec{\eta}(\tau, \lambda)$ inside the Wigner hyper-plane. Since $s=s(\tau, \lambda)$ is the arc-length along the string, the FS formulas are

$$
\begin{aligned}
& \frac{\partial_{\lambda} \hat{t}(\tau, \lambda)}{\partial_{\lambda} s(\tau, \lambda)}=\rho_{1}(\tau, \lambda) \hat{n}(\tau, \lambda) \\
& \frac{\partial_{\lambda} \hat{n}(\tau, \lambda)}{\partial_{\lambda} s(\tau, \lambda)}=-\rho_{1}(\tau, \lambda) \hat{t}(\tau, \lambda)+\rho_{2}(\tau, \lambda) \hat{b}(\tau, \lambda) \\
& \frac{\partial_{\lambda} \hat{b}(\tau, \lambda)}{\partial_{\lambda} s(\tau, \lambda)}=-\rho_{2}(\tau, \lambda) \hat{n}(\tau, \lambda),
\end{aligned}
$$

where $\rho_{1}(\tau, \lambda)$ is the curvature of the string and $\rho_{2}(\tau, \lambda)$ its torsion. These two quantities are Wigner 3-scalars and therefore Lorentz scalars.

The tangent vector, defined in Eq.(5.1), can be parametrized with two angles $\theta(\tau, \lambda)$, $\phi(\tau, \lambda)$, allowing one to find the following ortho-normal triad $\left(\hat{t}(\tau, \lambda), \hat{b}_{\theta}(\tau, \lambda), \hat{b}_{\phi}(\tau, \lambda)\right)$

$$
\begin{aligned}
\hat{t}(\tau, \lambda) & =-\frac{\vec{y}(\tau, \lambda)}{h(\tau, \lambda)} \stackrel{\text { def }}{=}(\sin \theta \cos \phi(\tau, \lambda), \sin \theta \sin \phi, \cos \theta)(\tau, \lambda), \\
\hat{b}_{\theta}(\tau, \lambda) & =\frac{\partial \hat{t}(\tau, \lambda)}{\partial \theta}=(\cos \theta \cos \phi, \cos \theta \sin \phi,-\sin \theta)(\tau, \lambda), \\
\hat{b}_{\phi}(\tau, \lambda) & =\left(\frac{1}{\sin \theta} \frac{\partial \hat{t}}{\partial \phi}\right)(\tau, \lambda)=(-\sin \phi, \cos \phi, 0)(\tau, \lambda),
\end{aligned}
$$

The first FS equation becomes ${ }^{9}$

$$
\begin{aligned}
& \partial_{\lambda} \hat{t}(\tau, \lambda)=\partial_{\lambda} s(\tau, \lambda) \rho_{1}(\tau, \lambda) \hat{n}(\tau, \lambda)= \\
= & \left(\cos \theta \cos \phi \partial_{\lambda} \theta-\sin \theta \sin \phi \partial_{\lambda} \phi, \cos \theta \sin \phi \partial_{\lambda} \theta+\sin \theta \cos \phi \partial_{\lambda} \phi,-\sin \theta \partial_{\lambda} \theta\right)(\tau, \lambda)= \\
= & {\left[\frac{\partial \hat{t}}{\partial \theta} \partial_{\lambda} \theta+\frac{\partial \hat{t}}{\partial \phi} \partial_{\lambda} \phi\right](\tau, \lambda)=\left[\partial_{\lambda} \theta \hat{b}_{\theta}+\sin \theta \partial_{\lambda} \phi \hat{b}_{\phi}\right](\tau, \lambda), }
\end{aligned}
$$

\footnotetext{
${ }^{9}$ We also have $\partial_{\lambda} \hat{b}_{\theta}(\tau, \lambda)=\left[-\partial_{\lambda} \theta \hat{t}+\cos \theta \partial_{\lambda} \phi \hat{b}_{\phi}\right](\tau, \lambda)$ and $\partial_{\lambda} \hat{b}_{\phi}(\tau, \lambda)=-\left[\partial_{\lambda} \phi\left(\sin \theta \hat{t}+\cos \theta \hat{b}_{\theta}\right)\right](\tau, \lambda)$.
} 


$$
\begin{gathered}
\Downarrow \\
\rho_{1}(\tau, \lambda)=\frac{1}{\partial_{\lambda} s(\tau, \lambda)} \sqrt{\left(\partial_{\lambda} \hat{t}(\tau, \lambda)\right)^{2}}=\frac{1}{\partial_{\lambda} s(\tau, \lambda)} \sqrt{\left(\partial_{\lambda} \theta\right)^{2}+\sin ^{2} \theta\left(\partial_{\lambda} \phi\right)^{2}}(\tau, \lambda) \\
\hat{n}(\tau, \lambda)=\frac{\left[\partial_{\lambda} \theta \hat{b}_{\theta}+\sin \theta \partial_{\lambda} \phi \hat{b}_{\phi}\right](\tau, \lambda)}{\partial_{\lambda} s(\tau, \lambda) \rho_{1}(\tau, \lambda)}=\frac{\left[\partial_{\lambda} \theta \hat{b}_{\theta}+\sin \theta \partial_{\lambda} \phi \hat{b}_{\phi}\right]}{\sqrt{\left(\partial_{\lambda} \theta\right)^{2}+\sin ^{2} \theta\left(\partial_{\lambda} \phi\right)^{2}}}(\tau, \lambda)
\end{gathered}
$$

Moreover we have

$$
\begin{aligned}
\hat{b}(\tau, \lambda)= & \hat{t}(\tau, \lambda) \times \hat{n}(\tau, \lambda)=\frac{\left[\partial_{\lambda} \theta \hat{b}_{\phi}-\sin \theta \partial_{\lambda} \phi \hat{b}_{\theta}\right]}{\sqrt{\left(\partial_{\lambda} \theta\right)^{2}+\sin ^{2} \theta\left(\partial_{\lambda} \phi\right)^{2}}}(\tau, \lambda) \\
\Downarrow & {\left[\left(\partial_{\lambda} \theta\right)^{2}+\sin ^{2} \theta\left(\partial_{\lambda} \phi\right)^{2}\right]^{3 / 2} } \\
\partial_{\lambda} \hat{b}(\tau, \lambda)= & \frac{\sin \theta\left(\partial_{\lambda} \phi \partial_{\lambda}^{2} \theta-\partial_{\lambda} \theta \partial_{\lambda}^{2} \phi\right)-\sin ^{2} \theta \cos \theta\left(\partial_{\lambda} \phi\right)^{3}-2 \cos \theta\left(\partial_{\lambda} \theta\right)^{2} \partial_{\lambda} \phi}{\left[\partial_{\lambda} \theta \hat{b}_{\theta}+\sin \theta \partial_{\lambda} \phi \hat{b}_{\phi}\right](\tau, \lambda)}(\tau, \lambda) \times \\
& \Downarrow \quad \frac{1}{\rho_{2}(\tau, \lambda)=} \\
= & \frac{\sin \theta\left(\partial_{\lambda} \phi \partial 2_{\lambda} \theta-\partial_{\lambda} \theta \partial_{\lambda}^{2} \phi\right)-\sin ^{2} \theta \cos \theta\left(\partial_{\lambda} \phi\right)^{3}-2 \cos \theta\left(\partial_{\lambda} \theta\right)^{2} \partial_{\lambda} \phi}{\partial_{\lambda} s\left[\left(\partial_{\lambda} \theta\right)^{2}+\sin ^{2} \theta\left(\partial_{\lambda} \phi\right)^{2}\right]}(\tau, \lambda) .
\end{aligned}
$$

Let us remark that the tangents at the end points are determined by $\vec{y}(\tau, 0 / \pi)=$ $-\left.\partial_{\lambda} s(\tau, \lambda)\right|_{\lambda=o / \pi} \hat{t}(\tau, 0 / \pi)$. Therefore, we have $\int_{o}^{\pi} d \lambda \partial_{\lambda} \vec{y}(\tau, \lambda)=\vec{y}(\tau, \pi)-\vec{y}(\tau, 0)$, $\int_{o}^{\tau} d \lambda \partial_{\lambda}^{2} \vec{y}(\tau \lambda)=\left.\partial_{\lambda} \vec{y}(\tau, \lambda)\right|_{\lambda=\pi}-\left.\partial_{\lambda} \vec{y}(\tau, \lambda)\right|_{\lambda=0}$.

Let us also remark that the negative parity of the variable $\vec{y}(\tau, \lambda)=-\vec{y}(\tau,-\lambda)$ implies $\hat{t}(\tau,-\lambda)=-\hat{t}(\tau, \lambda)$, so that from Eqs. (5.4) we get $\cos \theta(\tau,-\lambda)=-\cos \theta(\tau, \lambda)$. But this implies $\theta(\tau,-\lambda)=\theta(\tau, \lambda)+\pi$. Due to these discontinuities the description using the angles $\theta(\tau, \lambda)$ and $\phi(\tau, \lambda)$ must be restricted to the interval $(0, \pi)$, so that the distributions $\triangle_{ \pm}\left(\lambda, \lambda^{\prime}\right)$ will be restricted to $\delta\left(\lambda-\lambda^{\prime}\right)$ from now on.

The previous geometrical description of the string suggests to replace the canonical basis of relative variables $\vec{y}(\tau, \lambda), \overrightarrow{\mathcal{P}}(\tau, \lambda)$, with a new canonical basis defined by the following point canonical transformation 


$$
\begin{aligned}
& \begin{array}{|l|l|l|l|}
\hline \vec{y}(\tau, \lambda) \\
\hline \overrightarrow{\mathcal{P}}(\tau, \lambda) \\
\hline
\end{array} \longrightarrow \begin{array}{|l|l|l|}
\hline s(\tau, \lambda) & \theta(\tau, \lambda) & \phi(\tau, \lambda) \\
\hline \pi_{s}(\tau, \lambda) & \pi_{\theta}(\tau, \lambda) & \pi_{\phi}(\tau, \lambda) \\
\hline
\end{array} \\
& \left\{s(\tau, \lambda), \pi_{s}\left(\tau, \lambda_{1}\right)\right\}=\left\{\theta(\tau, \lambda), \pi_{\theta}\left(\tau, \lambda_{1}\right)\right\}=\left\{\phi(\tau, \lambda), \pi_{\phi}\left(\tau, \lambda_{1}\right)\right\}=\delta\left(\lambda-\lambda_{1}\right) .
\end{aligned}
$$

The point canonical transformation (5.7) (the old momenta are linearly connected to the new one) has the following generating function

$$
\begin{aligned}
\Phi & =\int d \lambda \mathcal{P}^{r}(\tau, \lambda)\left(-\partial_{\lambda} s(\tau, \lambda) \hat{t}^{r}(\tau, \lambda)\right)= \\
& =-\int d \lambda \partial_{\lambda} s(\tau, \lambda)\left[\mathcal{P}^{1} \sin \theta \cos \phi+\mathcal{P}^{2} \sin \theta \sin \phi+\mathcal{P}^{3} \cos \theta\right](\tau, \lambda)
\end{aligned}
$$

The equations

$$
\begin{aligned}
& \pi_{s}(\tau, \lambda)=\frac{\delta \Phi}{\delta s(\tau, \lambda)} \\
& \pi_{\theta}(\tau, \lambda)=\frac{\delta \Phi}{\delta \theta(\tau, \lambda)} \\
& \pi_{\phi}(\tau, \lambda)=\frac{\delta \Phi}{\delta \phi(\tau, \lambda)}
\end{aligned}
$$

imply

$$
\begin{aligned}
& \pi_{s}=\partial_{\lambda}(\overrightarrow{\mathcal{P}} \cdot \hat{t}) \\
& \pi_{\theta}=-\mathcal{P}^{1}\left(\partial_{\lambda} s\right) \cos \theta \cos \phi-\mathcal{P}^{2}\left(\partial_{\lambda} s\right) \cos \theta \sin \phi+\mathcal{P}^{3} \sin \theta=-\partial_{\lambda} s\left(\overrightarrow{\mathcal{P}} \cdot \hat{b}_{\theta}\right), \\
& \pi_{\phi}=\mathcal{P}^{1}\left(\partial_{\lambda} s\right) \sin \theta \sin \phi-\mathcal{P}^{2}\left(\partial_{\lambda} s\right) \sin \theta \cos \phi=-\partial_{\lambda} s \sin \theta\left(\overrightarrow{\mathcal{P}} \cdot \hat{b}_{\phi}\right),
\end{aligned}
$$

so that the expression of the old momenta in terms of the new ones is

$$
\overrightarrow{\mathcal{P}}(\tau, \lambda)=\hat{t}(\tau, \lambda) \int_{o}^{\lambda} d \lambda_{1} \pi_{s}\left(\tau, \lambda_{1}\right)-\frac{1}{\partial_{\lambda} s}\left[\pi_{\theta} \hat{b}_{\theta}+\frac{\pi_{\phi}}{\sin \theta} \hat{b}_{\phi}\right](\tau, \lambda) .
$$

We have the following relation between $\pi_{s}$ and the Abelianized constraint $\pi_{\chi}$ 


$$
\begin{aligned}
\pi_{\chi}(\tau, \lambda)= & {\left[\partial_{\lambda} \overrightarrow{\mathcal{P}} \cdot \hat{t}\right](\tau, \lambda)=\left[\pi_{s}-\partial_{\lambda} \theta \overrightarrow{\mathcal{P}} \cdot \hat{b}_{\theta}-\sin \theta \partial_{\lambda} \phi \overrightarrow{\mathcal{P}} \cdot \hat{b}_{\phi}\right](\tau \lambda)=} \\
= & {\left[\pi_{s}+\frac{\partial_{\lambda} \theta \pi_{\theta}+\partial_{\lambda} \phi \pi_{\phi}}{\partial_{\lambda} s}\right](\tau, \lambda), } \\
& \left\{\theta(\tau, \lambda), \pi_{\chi}\left(\tau, \lambda_{1}\right)\right\}=\frac{\partial_{\lambda} \theta}{\partial_{\lambda} s}(\tau, \lambda) \delta\left(\lambda-\lambda_{1}\right), \\
& \left\{\phi(\tau, \lambda), \pi_{\chi}\left(\tau, \lambda_{1}\right)\right\}=\frac{\partial_{\lambda} \phi}{\partial_{\lambda} s}(\tau, \lambda) \delta\left(\lambda-\lambda_{1}\right) .
\end{aligned}
$$

As a consequence, the functions $\theta(\tau, \lambda)$ and $\phi(\tau, \lambda)$ are not gauge invariant. To find the two corresponding Dirac observables we have to solve the following multi-temporal equations (generated by the constraint $\chi(\tau, \lambda) \approx 0)$, which determine the dependence of $\theta(\tau, \lambda)$ and $\phi(\tau, \lambda)$ on the gauge variable $s(\tau, \lambda)$

$$
\begin{aligned}
& \partial_{\lambda} s(\tau, \lambda) \frac{\delta \theta(\tau, \lambda)}{\delta s\left(\tau, \lambda_{1}\right)} \stackrel{\text { def }}{=}\left\{\theta(\tau, \lambda), \chi\left(\tau, \lambda_{1}\right)\right\}=\frac{\partial \theta(\tau, \lambda)}{\partial \lambda} \delta\left(\lambda-\lambda_{1}\right), \\
& \partial_{\lambda} s(\tau, \lambda) \frac{\delta \phi(\tau, \lambda)}{\delta s\left(\tau, \lambda_{1}\right)} \stackrel{\text { def }}{=}\left\{\phi(\tau, \lambda), \chi\left(\tau, \lambda_{1}\right)\right\}=\frac{\partial \phi(\tau, \lambda)}{\partial \lambda} \delta\left(\lambda-\lambda_{1}\right) .
\end{aligned}
$$

If we introduce the following ansatz

$$
\begin{gathered}
F(\tau, \lambda)=\bar{F}(\tau, s(\tau, \lambda))=T_{\lambda \mapsto s(\tau, \lambda)} \bar{F}(\tau, \lambda), \quad F=\theta, \phi \\
\Rightarrow \quad\left\{\bar{\theta}(\tau, \lambda), s\left(\tau, \lambda_{1}\right)\right\}=\left\{\bar{\phi}(\tau, \lambda), s\left(\tau, \lambda_{1}\right)\right\}=0, \\
T_{\lambda \mapsto s(\tau, \lambda)}=\sum_{o}^{\infty} \frac{1}{n !}(s(\tau, \lambda)-\lambda)^{n} \frac{\partial^{n}}{\partial \lambda^{n}},
\end{gathered}
$$

and we remember the results $\chi(\tau, \lambda)=\partial_{\lambda} s(\tau, \lambda) \pi_{\chi}(\tau, \lambda)$ and $\left\{s(\tau, \lambda), \chi\left(\tau, \lambda_{1}\right)\right\}=$ $\partial_{\lambda} s(\tau, \lambda) \delta\left(\lambda-\lambda_{1}\right)$, then we have 


$$
\begin{aligned}
& \partial_{\lambda} s(\tau, \lambda) \frac{\delta \bar{\theta}(\tau, s(\tau, \lambda))}{\delta s\left(\tau, \lambda_{1}\right)}=\left\{\bar{\theta}(\tau, s(\tau, \lambda)), \chi\left(\tau, \lambda_{1}\right)\right\}= \\
= & \partial_{\lambda} s(\tau, \lambda) \delta\left(\lambda-\lambda_{1}\right) T_{\lambda \mapsto s(\tau, \lambda)} \frac{\partial \bar{\theta}(\tau, \lambda)}{\partial \lambda}+T_{\lambda \mapsto s(\tau, \lambda)}\left\{\bar{\theta}(\tau, \lambda), \chi\left(\tau, \lambda_{1}\right)\right\}= \\
= & \frac{\partial \theta(\tau, \lambda)}{\partial \lambda} \delta\left(\lambda-\lambda_{1}\right)+T_{\lambda \mapsto s(\tau, \lambda)}\left\{\bar{\theta}(\tau, \lambda), \chi\left(\tau, \lambda_{1}\right)\right\}, \\
& \partial_{\lambda} s(\tau, \lambda) \frac{\delta \bar{\phi}(\tau, s(\tau, \lambda))}{\delta s\left(\tau, \lambda_{1}\right)}=\left\{\bar{\phi}(\tau, s(\tau, \lambda)), \chi\left(\tau, \lambda_{1}\right)\right\}= \\
= & \partial_{\lambda} s(\tau, \lambda) \delta\left(\lambda-\lambda_{1}\right) T_{\lambda \mapsto s(\tau, \lambda)} \frac{\partial \bar{\phi}(\tau, \lambda)}{\partial \lambda}+T_{\lambda \mapsto s(\tau, \lambda)}\left\{\bar{\phi}(\tau, \lambda), \chi\left(\tau, \lambda_{1}\right)\right\}= \\
= & \frac{\partial \phi(\tau, \lambda)}{\partial \lambda} \delta\left(\lambda-\lambda_{1}\right)+T_{\lambda \mapsto s(\tau, \lambda)}\left\{\bar{\phi}(\tau, \lambda), \chi\left(\tau, \lambda_{1}\right)\right\}, \\
& \Downarrow \\
& \left\{\bar{\theta}(\tau, \lambda), \chi\left(\tau, \lambda_{1}\right)\right\}=\left\{\bar{\phi}(\tau, \lambda), \chi\left(\tau, \lambda_{1}\right)\right\}=0 .
\end{aligned}
$$

Therefore a possible set of configurational Dirac observables for the open Nambu string is

$$
\begin{aligned}
& \bar{\theta}(\tau, \lambda)=T_{\lambda \mapsto s(\tau, \lambda)}^{-1} \theta(\tau, \lambda), \\
& \bar{\phi}(\tau, \lambda)=T_{\lambda \mapsto s(\tau, \lambda)}^{-1} \phi(\tau, \lambda) .
\end{aligned}
$$

As a consequence, after the canonical transformation (5.7) we must do another Shanmugadhasan point canonical transformation leading to a canonical basis adapted to the Abelianized constrains $\pi_{\chi}(\tau, \lambda) \approx 0$ and containing a canonical basis of Dirac observables for the open string

$$
\begin{aligned}
& \begin{array}{|l|l|l|l|l|l|}
\hline s(\tau, \lambda) & \theta(\tau, \lambda) & \phi(\tau, \lambda) \\
\hline \pi_{s}(\tau, \lambda) & \pi_{\theta}(\tau, \lambda) & \pi_{\phi}(\tau, \lambda)
\end{array} \longrightarrow \begin{array}{|l|l|l|}
\hline s(\tau, \lambda) & \bar{\theta}(\tau, \lambda) & \bar{\phi}(\tau, \lambda) \\
\hline \pi_{\chi}(\tau, \lambda) \approx 0 & \pi_{\bar{\theta}}(\tau, \lambda) & \bar{\pi}_{\bar{\phi}}(\tau, \lambda) \\
\hline
\end{array} \\
& \left\{s(\tau, \lambda), \pi_{\chi}\left(\tau, \lambda_{1}\right)\right\}=\left\{\bar{\theta}(\tau, \lambda), \pi_{\bar{\theta}}\left(\tau, \lambda_{1}\right)\right\}=\left\{\bar{\phi}(\tau, \lambda), \pi_{\bar{\phi}}\left(\tau, \lambda_{1}\right)\right\}=\delta\left(\lambda-\lambda_{1}\right) .
\end{aligned}
$$

The generating function of the point Shanmugadhasan canonical transformation (5.17) is 


$$
\begin{aligned}
\Phi_{1} & =\int d \lambda\left[\pi_{s} s+\pi_{\theta} T_{\lambda \mapsto s(\tau, \lambda)} \bar{\theta}+\pi_{\phi} T_{\lambda \mapsto s(\tau, \lambda)} \bar{\phi}\right](\tau, \lambda)= \\
& =\int d \lambda\left[\left(\pi_{s} s\right)(\tau, \lambda)+\pi_{\theta}(\tau, \lambda) \bar{\theta}(\tau, s(\tau, \lambda))+\pi_{\phi}(\tau, \lambda) \bar{\phi}(\tau, s(\tau, \lambda))\right] \\
\pi_{\chi}(\tau, \lambda) & =\frac{\delta \Phi_{1}}{\delta s(\tau, \lambda)} \\
\pi_{\bar{\theta}}(\tau, \lambda) & =\frac{\delta \Phi_{1}}{\delta \bar{\theta}(\tau, \lambda)} \\
\pi_{\bar{\phi}}(\tau, \lambda) & =\frac{\delta \Phi_{1}}{\delta \bar{\phi}(\tau, \lambda)} .
\end{aligned}
$$

The explicit expression of the new momenta is ${ }^{10}$

$$
\begin{aligned}
\pi_{\chi}(\tau, \lambda)= & \pi_{s}(\tau, \lambda)+\pi_{\theta}(\tau, \lambda) \partial_{s} \bar{\theta}(\tau, s(\tau, \lambda))+\pi_{\phi}(\tau, \lambda) \partial_{s} \bar{\phi}(\tau, s(\tau, \lambda))= \\
= & {\left[\pi_{s}+\frac{\partial_{\lambda} \theta \pi_{\theta}+\partial_{\lambda} \phi \pi_{\phi}}{\partial_{\lambda} s}\right](\tau, \lambda) \approx 0, } \\
\pi_{\bar{\theta}}(\tau, \lambda)= & \int d \lambda_{1} \pi_{\theta}\left(\tau, \lambda_{1}\right) T_{\lambda_{1} \mapsto s\left(\tau, \lambda_{1}\right)} \delta\left(\lambda-\lambda_{1}\right)= \\
= & \int d \lambda_{1} \pi_{\theta}\left(\tau, \lambda_{1}\right) \delta\left(\lambda-s\left(\tau, \lambda_{1}\right)\right)=\left(\left.\partial_{\bar{\lambda}} s(\tau, \bar{\lambda})\right|_{\bar{\lambda}=s^{-1}(\tau, \lambda)}\right)^{-1} \pi_{\theta}\left(\tau, s^{-1}(\tau, \lambda)\right), \\
\pi_{\bar{\phi}}(\tau, \lambda)= & \int d \lambda_{1} \pi_{\phi}\left(\tau, \lambda_{1}\right) T_{\lambda_{1} \mapsto s\left(\tau, \lambda_{1}\right)} \delta\left(\lambda-\lambda_{1}\right)= \\
= & \int d \lambda_{1} \pi_{\phi}\left(\tau, \lambda_{1}\right) \delta\left(\lambda-s\left(\tau, \lambda_{1}\right)\right)=\left(\left.\partial_{\bar{\lambda}} s(\tau, \bar{\lambda})\right|_{\bar{\lambda}=s^{-1}(\tau, \lambda)}\right)^{-1} \pi_{\phi}\left(\tau, s^{-1}(\tau, \lambda)\right), \\
& \Downarrow
\end{aligned}
$$

10 We use $\theta(\tau, \lambda)=\bar{\theta}(\tau, s(\tau, \lambda)), \quad \phi(\tau, \lambda)=\bar{\phi}(\tau, s(\tau, \lambda))$ and then we have $\hat{t}(\tau \lambda)=$ $\hat{\bar{t}}(\tau, s(\tau, \lambda)), \quad \hat{b}_{\theta}(\tau \lambda)=\hat{\bar{b}}_{\bar{\theta}}(\tau, s(\tau, \lambda)), \quad \hat{b}_{\phi}(\tau \lambda)=\hat{\bar{b}}_{\bar{\phi}}(\tau, s(\tau, \lambda))$. We use $\partial_{s} \bar{\theta}(\tau, s(\tau, \lambda))=$ $\partial_{\lambda} \theta(\tau, \lambda) / \partial_{\lambda} s(\tau, \lambda)$, the analogous formula for $\bar{\phi}$ and $\delta\left(\lambda-s\left(\tau, \lambda_{1}\right)\right)=\delta\left(\lambda_{1}-\right.$ $\left.s^{-1}(\tau, \lambda)\right) /\left|\partial_{\lambda_{1}} s\left(\tau, \lambda_{1}\right)\right|_{\lambda_{1}=s^{-1}(\tau, \lambda)}$. 


$$
\begin{aligned}
\pi_{\theta}(\tau, \lambda) & =\partial_{\lambda} s(\tau, \lambda) \pi_{\bar{\theta}}(\tau, s(\tau, \lambda)) \\
\pi_{\phi}(\tau, \lambda) & =\partial_{\lambda} s(\tau, \lambda) \pi_{\bar{\phi}}(\tau, s(\tau, \lambda)) \\
\pi_{s}(\tau, \lambda) & =\pi_{\chi}(\tau, \lambda)-\partial_{\lambda} s(\tau, \lambda)\left[\partial_{s} \bar{\theta} \pi_{\bar{\theta}}+\partial_{s} \bar{\phi} \pi_{\bar{\phi}}\right](\tau, s(\tau, \lambda))= \\
& =\pi_{\chi}(\tau, \lambda)-\left[\partial_{\lambda} \bar{\theta} \pi_{\bar{\theta}}+\partial_{\lambda} \bar{\phi} \pi_{\bar{\phi}}\right](\tau, s(\tau, \lambda)), \\
\vec{y}(\tau, \lambda) & =-\partial_{\lambda} s(\tau, \lambda) \hat{\bar{t}}(\tau, s(\tau, \lambda)), \\
\overrightarrow{\mathcal{P}}(\tau, \lambda) & \left.=\hat{\bar{t}}_{(\tau,} s(\tau, \lambda)\right) \int_{o}^{\lambda} d \lambda_{1}\left[\pi_{\chi}\left(\tau, \lambda_{1}\right)-\left(\partial_{\lambda_{1}} \bar{\theta} \pi_{\bar{\theta}}+\partial_{\lambda_{1}} \bar{\phi} \pi_{\bar{\phi}}\right)\left(\tau, s\left(\tau, \lambda_{1}\right)\right)\right]- \\
& -\left[\pi_{\bar{\theta}} \hat{\bar{b}}_{\bar{\theta}}+\frac{\pi_{\bar{\phi}}}{\sin \bar{\theta}} \hat{\bar{b}}_{\bar{\phi}}\right](\tau, s(\tau, \lambda)) .
\end{aligned}
$$

The invariant mass becomes

$$
\begin{aligned}
& M=\int_{o}^{\pi} d \lambda \sqrt{-N^{2}\left(\partial_{\lambda} s(\tau, \lambda)\right)^{2}+\left(\partial_{\lambda} \overrightarrow{\mathcal{P}}\right)^{2}(\tau, \lambda)}, \\
& \partial_{\lambda} \overrightarrow{\mathcal{P}}(\tau, \lambda) \approx \hat{\bar{t}}(\tau, s(\tau, \lambda)) \pi_{\chi}(\tau, \lambda)+ \\
& +\hat{\bar{b}}_{\bar{\theta}}\left[-\partial_{\lambda} \bar{\theta} \int_{o}^{\lambda} d \lambda_{1}\left(\partial_{\lambda_{1}} \bar{\theta} \pi_{\bar{\theta}}+\partial_{\lambda_{1}} \bar{\phi} \pi_{\bar{\phi}}\right)\left(\tau, s\left(\tau, \lambda_{1}\right)\right)\right. \\
& \left.-\partial_{\lambda} \pi_{\bar{\theta}}+\frac{\cos \bar{\theta}}{\sin \bar{\theta}} \pi_{\bar{\phi}} \partial_{\lambda} \bar{\phi}\right](\tau, s(\tau, \lambda))+ \\
& +\hat{\bar{b}}_{\bar{\phi}}\left[-\sin \bar{\theta} \partial_{\lambda} \bar{\phi} \int_{o}^{\lambda} d \lambda_{1}\left(\partial_{\lambda_{1}} \bar{\theta} \pi_{\bar{\theta}}+\partial_{\lambda_{1}} \bar{\phi} \pi_{\bar{\phi}}\right)\left(\tau, s\left(\tau, \lambda_{1}\right)\right)\right. \\
& \left.-\frac{\partial_{\lambda} \pi_{\bar{\phi}}}{\sin \bar{\theta}}+\frac{\cos \bar{\theta}}{\sin 2 \bar{\theta}} \pi_{\bar{\phi}} \partial_{\lambda} \bar{\theta}-\cos \bar{\theta} \pi_{\bar{\theta}} \partial_{\lambda} \bar{\phi}\right](\tau, s(\tau, \lambda))
\end{aligned}
$$

We can now restrict ourselves to the FS gauge $s(\tau, \lambda) \approx \lambda$, which identifies the parameter $\lambda$ with the arc-length along the open string. In this way the open string is completely described in terms of the external center of mass (the frozen Jacobi data $\vec{z}$ and $\vec{h}$ ) and of the Dirac observables $\bar{\theta}, \pi_{\bar{\theta}}, \bar{\phi}, \pi_{\bar{\phi}}$. In the FS gauge we get (the Fokker-Pryce center of inertia $Y^{\mu}(\tau)$ is given in Eq. $\left.(4.2)\right)$ 


$$
\begin{aligned}
& x^{\mu}(\tau, \lambda)=Y^{\mu}(\tau)+\epsilon_{r}^{\mu}(\vec{h}) \eta^{r}(\tau, \lambda), \\
& \vec{\eta}(\tau) \approx+\frac{1}{2 \pi \int_{o}^{\pi} d \lambda \sqrt{-N^{2}+\left(\partial_{\lambda} \overrightarrow{\mathcal{P}}(\tau, \lambda)\right)^{2}}} \\
& \int_{o}^{\pi} d \lambda \int_{-\pi}^{\pi} d \lambda_{1} \int_{\lambda}^{\lambda_{1}} \vec{y}\left(\tau, \lambda_{2}\right) \sqrt{-N^{2}+\left(\partial_{\lambda} \overrightarrow{\mathcal{P}}(\tau, \lambda)\right)^{2}} \\
& \vec{y}(\tau, \lambda)=-\hat{\bar{t}}(\tau, \lambda)=-(\sin \bar{\theta} \cos \bar{\phi}, \sin \bar{\theta} \sin \bar{\phi}, \cos \bar{\theta})(\tau, \lambda),
\end{aligned}
$$

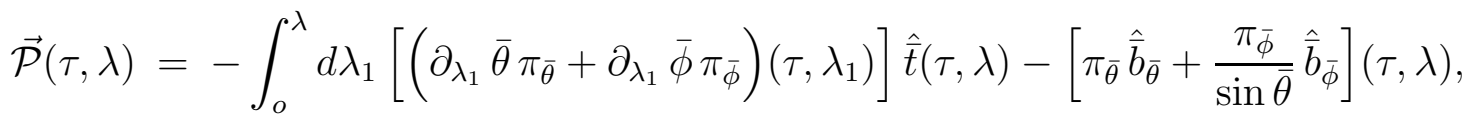

$$
\begin{aligned}
& M=\int_{0}^{\pi} d \lambda \sqrt{-N^{2}+A^{2}(\tau, \lambda)+B^{2}(\tau, \lambda)} \\
& \partial_{\lambda} \overrightarrow{\mathcal{P}}(\tau, \lambda)=\hat{\bar{b}}_{\bar{\theta}}(\tau, \lambda) A(\tau, \lambda)+\hat{\bar{b}}_{\bar{\phi}}(\tau, \lambda) B(\tau, \lambda) \\
& A(\tau, \lambda)=-\partial_{\lambda} \bar{\theta}(\tau, \lambda) \int_{o}^{\lambda} d \lambda_{1}\left(\partial_{\lambda_{1}} \bar{\theta} \pi_{\bar{\theta}}+\partial_{\lambda_{1}} \bar{\phi} \pi_{\bar{\phi}}\right)\left(\tau, \lambda_{1}\right)- \\
& -\partial_{\lambda} \pi_{\bar{\theta}}(\tau, \lambda)+\left[\frac{\cos \bar{\theta}}{\sin \bar{\theta}} \pi_{\bar{\phi}} \partial_{\lambda} \bar{\phi}\right](\tau, \lambda), \\
& B(\tau, \lambda)=-\left[\sin \bar{\theta} \partial_{\lambda} \bar{\phi}\right](\tau, \lambda) \int_{o}^{\lambda} d \lambda_{1}\left(\partial_{\lambda_{1}} \bar{\theta} \pi_{\bar{\theta}}+\partial_{\lambda_{1}} \bar{\phi} \pi_{\bar{\phi}}\right)\left(\tau, \lambda_{1}\right)- \\
& -\frac{\partial_{\lambda} \pi_{\bar{\phi}}(\tau, \lambda)}{\sin \bar{\theta}(\tau, \lambda)}+\left[\frac{\cos \bar{\theta}}{\sin 2 \bar{\theta}} \pi_{\bar{\phi}} \partial_{\lambda} \bar{\theta}-\cos \bar{\theta} \pi_{\bar{\theta}} \partial_{\lambda} \bar{\phi}\right](\tau, \lambda) \\
& \vec{S}=\int_{o}^{\pi} d \lambda\left[+\pi_{\bar{\theta}} \hat{\bar{b}}_{\bar{\phi}}-\frac{\pi_{\bar{\phi}}}{\sin \bar{\theta}} \hat{\bar{b}}_{\bar{\theta}}\right](\tau, \lambda), \\
& \rho_{1}(\tau, \lambda)=\sqrt{\left(\partial_{\lambda} \bar{\theta}\right)^{2}+\sin ^{2} \bar{\theta}\left(\partial_{\lambda} \bar{\phi}\right)^{2}}(\tau, \lambda), \\
& \rho_{2}(\tau, \lambda)=\frac{\sin \bar{\theta}\left(\partial_{\lambda} \bar{\phi} \partial_{\lambda}^{2} \bar{\theta}-\partial_{\lambda} \bar{\theta} \partial_{\lambda}^{2} \bar{\phi}\right)-\sin 2 \bar{\theta} \cos \bar{\theta}\left(\partial_{\lambda} \bar{\phi}\right)^{3}-2 \cos \bar{\theta}\left(\partial_{\lambda} \bar{\theta}\right)^{2} \partial_{\lambda} \bar{\phi}}{\left[\left(\partial_{\lambda} \bar{\theta}\right)^{2}+\sin ^{2} \bar{\theta}\left(\partial_{\lambda} \bar{\phi}\right)^{2}\right]}(\tau, \lambda) \text {. }
\end{aligned}
$$

where Eq.(4.9) was used.

The resulting Hamilton equations for the Dirac observables must be integrated with the boundary conditions $\dot{\vec{\eta}}_{o}^{2}(\tau)=\dot{\vec{\eta}}_{\pi}^{2}(\tau)=1\left(\dot{\vec{\eta}}_{A}(\tau)=\left.\partial_{\tau} \vec{\eta}(\tau, \lambda)\right|_{\lambda=A}, A=0, \pi\right)$, which imply that 
the end points of the string $\vec{\eta}_{A}(\tau)=\vec{\eta}(\tau, A), A=0, \pi$, move on null curves. Note that we have $\vec{\eta}_{o}(\tau)-\vec{\eta}_{\pi}(\tau)=\int_{o}^{\pi} d \lambda \hat{\bar{t}}(\tau, \lambda)$. Moreover we have $\hat{\bar{t}}_{A}(\tau)=\hat{\bar{t}}(\tau, A)=-\vec{y}(\tau, A), A=0, \pi$, with $\hat{\bar{t}}_{\pi}(\tau)-\hat{\bar{t}}_{o}(\tau)=\int_{o}^{\pi} d \lambda \partial_{\lambda} \hat{\bar{t}}(\tau, \lambda)$.

The Hamilton equations generated by the Hamiltonian $M$ are

$$
\begin{aligned}
& \partial_{\tau} \bar{\theta}(\tau, \lambda)=-\partial_{\lambda} \bar{\theta}(\tau, \lambda) \int_{\lambda}^{\pi} d \lambda^{\prime}\left[\frac{\partial_{\lambda} \bar{\theta} A+\sin \bar{\theta} \partial_{\lambda} \bar{\phi} B}{\sqrt{-N^{2}+A^{2}+B^{2}}}\right]\left(\tau, \lambda^{\prime}\right)+ \\
& +\partial_{\lambda}\left(\frac{A}{\sqrt{-N^{2}+A^{2}+B^{2}}}\right)(\tau, \lambda)-\left(\frac{\cos \bar{\theta} \partial_{\lambda} \bar{\phi} B}{\sqrt{-N^{2}+A^{2}+B^{2}}}\right)(\tau, \lambda), \\
& \partial_{\tau} \bar{\phi}(\tau, \lambda)=-\partial_{\lambda} \bar{\phi}(\tau, \lambda) \int_{\lambda}^{\pi} d \lambda^{\prime}\left[\frac{\partial_{\lambda} \bar{\theta} A+\sin \bar{\theta} \partial_{\lambda} \bar{\phi} B}{\sqrt{-N^{2}+A^{2}+B^{2}}}\right]\left(\tau, \lambda^{\prime}\right)+ \\
& +\partial_{\lambda}\left(\frac{B}{\sin \bar{\theta} \sqrt{-N^{2}+A^{2}+B^{2}}}\right)(\tau, \lambda)+\left(\frac{\frac{\cos \bar{\theta}}{\sin \theta} \partial_{\lambda} \bar{\phi} A-\frac{\cos \bar{\theta}}{\sin 2 \bar{\theta}} \partial_{\lambda} \bar{\theta} B}{\sqrt{-N^{2}+A^{2}+B^{2}}}\right)(\tau, \lambda), \\
& \partial_{\tau} \pi_{\bar{\theta}}(\tau, \lambda)=-\partial_{\lambda} \pi_{\bar{\theta}}(\tau, \lambda) \int_{\lambda}^{\pi} d \lambda^{\prime}\left[\frac{\partial_{\lambda} \bar{\theta} A+\sin \bar{\theta} \partial_{\lambda} \bar{\phi} B}{\sqrt{-N^{2}+A^{2}+B^{2}}}\right]\left(\tau, \lambda^{\prime}\right)+ \\
& +\partial_{\lambda}\left(\frac{\frac{\cos \bar{\theta}}{\sin \theta} \pi_{\bar{\phi}} B}{\sqrt{-N^{2}+A^{2}+B^{2}}}\right)(\tau, \lambda)+ \\
& -\left(\frac{\left(\pi_{\bar{\theta}} \partial_{\lambda} \bar{\theta}+\frac{\bar{\phi} \partial_{\lambda} \bar{\phi}}{\sin 2 \theta}\right) A+\left(\frac{\cos \bar{\theta}}{\sin \bar{\theta}} \partial_{\lambda} \pi_{\bar{\phi}}+\frac{1+\cos 2 \bar{\theta}}{\sin 2 \bar{\theta}} \pi_{\bar{\phi}} \partial_{\lambda} \bar{\theta}\right) B}{\sqrt{-N^{2}+A^{2}+B^{2}}}\right)(\tau, \lambda), \\
& \partial_{\tau} \pi_{\bar{\phi}}(\tau, \lambda)=-\partial_{\lambda} \pi_{\bar{\phi}}(\tau, \lambda) \int_{\lambda}^{\pi} d \lambda^{\prime}\left[\frac{\partial_{\lambda} \bar{\theta} A+\sin \bar{\theta} \partial_{\lambda} \bar{\phi} B}{\sqrt{-N^{2}+A^{2}+B^{2}}}\right]\left(\tau, \lambda^{\prime}\right)+ \\
& +\partial_{\lambda}\left(\frac{\frac{\cos \bar{\theta}}{\sin \theta} \pi_{\bar{\phi}} A+\cos \bar{\theta} \pi_{\bar{\theta}} B}{\sqrt{-N^{2}+A^{2}+B^{2}}}\right)(\tau, \lambda)+ \\
& -\left(\frac{\pi_{\bar{\phi}} \partial_{\lambda} \bar{\theta} A+\sin \bar{\theta} \partial_{\lambda} \bar{\phi} \pi_{\bar{\phi}} B}{\sqrt{-N^{2}+A^{2}+B^{2}}}\right)(\tau, \lambda) \text {. }
\end{aligned}
$$

This series of point canonical transformations should be completed with the following one 


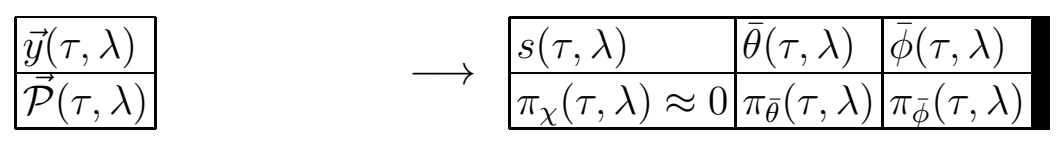

$$
\begin{aligned}
& \longrightarrow \begin{array}{|l|l|l|}
\hline s(\tau, \lambda) & \rho_{1}(\tau, \lambda) & \rho_{2}(\tau, \lambda) \\
\hline \pi_{\chi}(\tau, \lambda) \approx 0 & \pi_{1}(\tau, \lambda) & \pi_{2}(\tau, \lambda) \\
\hline
\end{array}
\end{aligned}
$$

in which the Dirac observables $\bar{\theta}, \pi_{\bar{\theta}}, \bar{\phi}, \pi_{\bar{\phi}}$, are replaced with the Lorentz scalar Dirac observables $\rho_{1}, \pi_{1}, \rho_{2}, \pi_{2}$ given in Eqs.(5.21). Since we have $\rho_{1}(\tau, \lambda) \geq 0$ and $\rho_{2}(\tau, \lambda) \geq 0$, these variables are like radial variables ${ }^{11}$.

Regarding the last canonical transformation (5.23), we know $\bar{\rho}_{i}=\bar{\rho}_{i}(\bar{\theta}, \bar{\phi}), i=1,2$, from Eqs. (5.21), but not the inversion $\bar{\theta}=\bar{\theta}\left(\bar{\rho}_{i}\right), \bar{\phi}=\bar{\phi}\left(\bar{\rho}_{i}\right)$. Therefore we can only define the generating function of the inverse canonical transformation

$$
\begin{aligned}
\Psi & =\int d \lambda\left[\bar{\pi}_{1} \bar{\rho}_{1}(\bar{\theta}, \bar{\phi})+\bar{\pi}_{2} \bar{\rho}_{2}(\bar{\theta}, \bar{\phi})\right](\tau, \lambda) \\
\pi_{\bar{\theta}}(\tau, \lambda) & =\frac{\delta \Psi}{\delta \bar{\theta}(\tau, \lambda)} \\
\pi_{\bar{\phi}}(\tau, \lambda) & =\frac{\delta \Psi}{\delta \bar{\phi}(\tau, \lambda)} .
\end{aligned}
$$

It can give $\bar{\pi}_{i}=\bar{\pi}_{i}\left(\bar{\theta}, \pi_{\bar{\theta}}, \bar{\phi}, \pi_{\bar{\phi}}\right)$, linear in $\pi_{\bar{\theta}}$ and $\pi_{\bar{\phi}}$.

However, to find the expression of the invariant mass $M$ we need the inversion $\bar{\theta}\left(\bar{\rho}_{i}\right)$, $\bar{\phi}\left(\bar{\rho}_{i}\right), \pi_{\bar{\theta}}\left(\bar{\rho}_{i}, \bar{\pi}_{i}\right), \pi_{\bar{\phi}}\left(\bar{\rho}_{i}, \bar{\pi}_{i}\right)^{12}$.

${ }^{11}$ In trying to quantize them one should use the non-canonical basis $r$ and $r p_{r}$, the only one which would give meaningful commutators due to $r \geq 0$.

12 The existence of the inversion is assured by theorem (7.5) in chapter 7 of Ref. [23]. It implies that, according to the fundamental theorem for curves, the embedding functions $\vec{\eta}(\tau, \lambda)$, and therefore $\vec{y}(\tau, \lambda)=\hat{\bar{t}}(\tau, \lambda)=$ $-\partial_{\lambda} \vec{\eta}(\tau, \lambda)$, can be obtained from the Frenet-Serret curvatures $\bar{\rho}_{i}(\tau, \lambda)$ by quadratures. 


\section{PARTICLE-LIKE VARIABLES FOR THE TRANSVERSE OSCILLATIONS AND THE PROBLEM OF QUANTIZATION.}

Since we cannot find explicitly the canonical transformation to the canonical basis of Dirac observables $\rho_{i}, \pi_{i}$, whose inverse is defined in Eq.(5.23), let us look for a different canonical basis of Dirac observables, which avoids the angles because they cannot be quantized consistently.

If we interpret the angles and their conjugate momenta as angle-action-like variables, the following canonical transformation emerges in a natural way $\left[N / c\right.$ has dimensions $m t^{-1}$ like $m \omega$ in the harmonic oscillator $H=\frac{p^{2}}{2 m}+\frac{1}{2} m \omega^{2} q^{2}=\omega I$, with $I$ action variable]

$$
\begin{aligned}
q_{\bar{\theta}}(\tau, \lambda)= & +\sqrt{\frac{2 c}{N} \pi_{\bar{\theta}}(\tau, \lambda)} \cos \bar{\theta}(\tau, \lambda) \\
p_{\bar{\theta}}(\tau, \lambda)= & -\sqrt{\frac{2 N}{c} \pi_{\bar{\theta}}(\tau, \lambda)} \sin \bar{\theta}(\tau, \lambda) \\
q_{\bar{\phi}}(\tau, \lambda)= & +\sqrt{\frac{2 c}{N} \pi_{\bar{\phi}}(\tau, \lambda)} \cos \bar{\phi}(\tau, \lambda) \\
p_{\bar{\phi}}(\tau, \lambda)= & -\sqrt{\frac{2 N}{c} \pi_{\bar{\phi}}(\tau, \lambda) \sin \bar{\phi}(\tau, \lambda),} \\
& \left\{q_{\bar{\theta}}(\tau, \lambda), p_{\bar{\theta}}\left(\tau, \lambda_{1}\right)\right\}=\left\{q_{\bar{\phi}}(\tau, \lambda), p_{\bar{\phi}}\left(\tau, \lambda_{1}\right)\right\}=\delta\left(\lambda-\lambda_{1}\right), \\
& \sin \bar{\theta}(\tau, \lambda)=-\frac{p_{\bar{\theta}}}{\sqrt{\frac{N^{2}}{c^{2}} q_{\bar{\theta}}^{2}+p_{\bar{\theta}}^{2}}}(\tau, \lambda), \quad \cos \bar{\theta}(\tau, \lambda)=\frac{q_{\bar{\theta}}}{\sqrt{q_{\bar{\theta}}^{2}+\frac{c^{2}}{N^{2}} p_{\bar{\theta}}^{2}}}(\tau, \lambda), \\
& \pi_{\bar{\theta}}(\tau, \lambda)=\left(\frac{N}{2 c} q_{\bar{\theta}}^{2}+\frac{c}{2 N} p_{\bar{\theta}}^{2}\right)(\tau, \lambda), \quad \cos \bar{\phi}(\tau, \lambda)=\frac{q_{\bar{\phi}}}{\sqrt{q_{\bar{\phi}}^{2}+\frac{c^{2}}{N^{2}} p_{\bar{\phi}}^{2}}}(\tau, \lambda), \\
& \sin \bar{\phi}(\tau, \lambda)=-\frac{p_{\bar{\phi}}}{\sqrt{\frac{N^{2}}{c^{2}} q_{\bar{\phi}}^{2}+p_{\bar{\phi}}^{2}}}(\tau, \lambda), \\
& \pi_{\bar{\phi}}(\tau, \lambda)=\left(\frac{N}{2 c} q_{\bar{\phi}}^{2}+\frac{c}{2 N} p_{\bar{\phi}}^{2}\right)(\tau, \lambda) .
\end{aligned}
$$

The particle-like variables $q_{\bar{\theta}}(\tau, \lambda), p_{\bar{\theta}}(\tau, \lambda), q_{\bar{\phi}}(\tau, \lambda), p_{\bar{\phi}}(\tau, \lambda)$ describe the transverse oscillations of the Nambu string.

We get ${ }^{13}$

${ }^{13}$ We use

$$
\partial_{\lambda} \sin \bar{\theta}(\tau, \lambda)=\cos \bar{\theta}(\tau, \lambda) \partial_{\lambda} \bar{\theta}(\tau, \lambda) \Rightarrow \partial_{\lambda} \bar{\theta}(\tau, \lambda)=\frac{\partial_{\lambda} \sin \bar{\theta}(\tau, \lambda)}{\cos \bar{\theta}(\tau, \lambda)}
$$

and then we make the substitutions:

$$
\sin \bar{\theta}(\tau, \lambda)=-\frac{p_{\bar{\theta}}}{\sqrt{\frac{N^{2}}{c^{2}} q_{\bar{\theta}}^{2}+p_{\bar{\theta}}^{2}}}(\tau, \lambda), \quad \cos \bar{\theta}(\tau, \lambda)=\frac{q_{\bar{\theta}}}{\sqrt{q_{\bar{\theta}}^{2}+\frac{c^{2}}{N^{2}} p_{\bar{\theta}}^{2}}}(\tau, \lambda)
$$




$$
\begin{aligned}
\partial_{\lambda} \bar{\theta}(\tau, \lambda) & =\frac{p_{\bar{\theta}} \partial_{\lambda} q_{\bar{\theta}}-q_{\bar{\theta}} \partial_{\lambda} \bar{\theta}}{\frac{N}{c} q_{\bar{\theta}}^{2}+\frac{c}{N} p_{\bar{\theta}}^{2}}(\tau, \lambda), \\
\partial_{\lambda} \bar{\phi}(\tau, \lambda) & =\frac{p_{\bar{\phi}} \partial_{\lambda} q_{\bar{\phi}}-q_{\bar{\phi}} \partial_{\lambda} \bar{\phi}}{\frac{N}{c} q_{\bar{\phi}}^{2}+\frac{c}{N} p_{\bar{\phi}}^{2}}(\tau, \lambda),
\end{aligned}
$$

so that we have

$$
\begin{aligned}
& M=\int_{o}^{\pi} d \lambda \sqrt{-N^{2}+A^{2}(\tau, \lambda)+B^{2}(\tau, \lambda)}, \\
A(\tau, \lambda)= & -\frac{1}{2} \frac{p_{\bar{\theta}} \partial_{\lambda} q_{\bar{\theta}}-q_{\bar{\theta}} \partial_{\lambda} p_{\bar{\theta}}}{\frac{N}{c} q_{\bar{\theta}}^{2}+\frac{c}{N} p_{\bar{\theta}}^{2}}(\tau, \lambda) \int_{o}^{\lambda} d \lambda_{1}\left(p_{\bar{\theta}} \partial_{\lambda} q_{\bar{\theta}}-q_{\bar{\theta}} \partial_{\lambda} p_{\bar{\theta}}+p_{\bar{\phi}} \partial_{\lambda} q_{\bar{\phi}}-q_{\bar{\phi}} \partial_{\lambda} p_{\bar{\phi}}\right)\left(\tau, \lambda_{1}\right)- \\
- & {\left[\frac{N}{c} q_{\bar{\theta}} \partial_{\lambda} q_{\bar{\theta}}-\frac{c}{N} p_{\bar{\theta}} \partial_{\lambda} p_{\bar{\theta}}+\frac{N}{2 c} \frac{q_{\bar{\theta}}}{p_{\bar{\theta}}}\left(p_{\bar{\phi}} \partial_{\lambda} q_{\bar{\phi}}+q_{\bar{\phi}} \partial_{\lambda} p_{\bar{\phi}}\right)\right](\tau, \lambda), } \\
B(\tau, \lambda)= & {\left[\frac{p_{\bar{\theta}}}{2 \sqrt{\frac{N^{2}}{c^{2}} q_{\bar{\theta}}^{2}+p_{\bar{\theta}}^{2}}} \frac{p_{\bar{\phi}} \partial_{\lambda} q_{\bar{\phi}}-q_{\bar{\phi}} \partial_{\lambda} p_{\bar{\phi}}}{\frac{N}{c} q_{\bar{\phi}}^{2}+\frac{c}{N} p_{\bar{\phi}}^{2}}\right](\tau, \lambda) } \\
& \int_{o}^{\lambda} d \lambda_{1}\left(p_{\bar{\theta}} \partial_{\lambda} q_{\bar{\theta}}-q_{\bar{\theta}} \partial_{\lambda} p_{\bar{\theta}}+p_{\bar{\phi}} \partial_{\lambda} q_{\bar{\phi}}-q_{\bar{\phi}} \partial_{\lambda} p_{\bar{\phi}}\right)\left(\tau, \lambda_{1}\right)+ \\
+ & {\left[\frac{\sqrt{\frac{N^{2}}{c^{2}} q_{\bar{\theta}}^{2}+p_{\bar{\theta}}^{2}}}{p_{\bar{\theta}}}\left(\frac{N}{c} q_{\bar{\phi}} \partial_{\lambda} q_{\bar{\phi}}+\frac{c}{N} p_{\bar{\phi}} \partial_{\lambda} p_{\bar{\phi}}\right)+\frac{N}{c} \frac{q_{\bar{\theta}}}{p_{\bar{\theta}}^{2}}\left(\frac{N}{2 c} q_{\bar{\phi}}^{2}+\frac{c}{2 N} p_{\bar{\phi}}^{2}\right) \frac{p_{\bar{\theta}} \partial_{\lambda} q_{\bar{\theta}}-q_{\bar{\theta}} \partial_{\lambda} p_{\bar{\theta}}}{\sqrt{q_{\bar{\theta}}^{2}+\frac{c^{2}}{N^{2}} p_{\bar{\theta}}^{2}}}\right.} \\
- & \left.\frac{1}{2} q_{\bar{\theta}} \frac{p_{\bar{\phi}} \partial_{\lambda} q_{\bar{\phi}}-q_{\bar{\phi}} \partial_{\lambda} p_{\bar{\phi}}}{\frac{N}{c} q_{\bar{\phi}}^{2}+\frac{c}{N} p_{\bar{\phi}}^{2}} \sqrt{\frac{N^{2}}{c^{2}} q_{\bar{\theta}}^{2}+p_{\bar{\theta}}^{2}}\right](\tau, \lambda) .
\end{aligned}
$$

We get the following spin vector [non-linear realization of $\mathrm{O}(3)$ ]

$$
\begin{aligned}
\vec{S} & =\int_{o}^{\pi} d \lambda \vec{S}(\tau, \lambda), \\
S^{1}(\tau, \lambda) & =\left(\frac{p_{\bar{\phi}}\left(\frac{N}{2 c} q_{\bar{\theta}}^{2}+\frac{N}{2 c} p_{\bar{\theta}}^{2}\right)}{\sqrt{\frac{N^{2}}{c^{2}} q_{\bar{\phi}}^{2}+p_{\bar{\phi}}^{2}}}+\left(\frac{N}{c}\right)^{3 / 2} \frac{q_{\bar{\theta}} q_{\bar{\phi}} \sqrt{\frac{N}{c} q_{\bar{\phi}}^{2}+\frac{c}{N} p_{\bar{\phi}}^{2}}}{2 p_{\bar{\theta}}}\right)(\tau, \lambda), \\
S^{2}(\tau, \lambda) & =\left(\frac{q_{\bar{\phi}}\left(\frac{N}{2 c} q_{\bar{\theta}}^{2}+\frac{c}{2 N} p_{\bar{\theta}}^{2}\right)}{\sqrt{q_{\bar{\phi}}^{2}+\frac{c^{2}}{N^{2}} p_{\bar{\phi}}^{2}}}-\sqrt{\frac{N}{c}} \frac{q_{\bar{\theta}} p_{\bar{\phi}} \sqrt{\frac{N}{c} q_{\bar{\phi}}^{2}+\frac{c}{N} p_{\bar{\phi}}^{2}}}{2 p_{\bar{\theta}}}\right)(\tau, \lambda), \\
S^{3}(\tau, \lambda) & \left.=-\left(\frac{N}{2 c} q_{\bar{\phi}}^{2}+\frac{c}{2 N} p_{\bar{\phi}}^{2}\right)\right)(\tau, \lambda) .
\end{aligned}
$$


Eqs.(6.4) are well defined either if $q_{\bar{\theta}}(\tau, \lambda) \neq 0$ for every $\lambda$ or if $p_{\bar{\theta}}(\tau, \lambda) / q_{\bar{\theta}}(\tau, \lambda)$ tends to a finite limit for $q_{\bar{\theta}}(\tau, \lambda) \rightarrow 0$.

Finally we can introduce a canonical basis $a_{i}(\tau, \lambda), a_{i}^{*}(\tau, \lambda), i=1,2$, of quantities which are the classical background for two sets of creation and annihilation operators

$$
\begin{aligned}
& q_{\bar{\theta}}(\tau, \lambda)=\sqrt{\frac{c}{2 N}}\left(a_{1}+a_{1}^{*}\right)(\tau, \lambda), \quad p_{\bar{\theta}}(\tau, \lambda)=-i \sqrt{\frac{N}{2 c}}\left(a_{1}-a_{1}^{*}\right)(\tau, \lambda), \\
& q_{\bar{\phi}}(\tau, \lambda)=\sqrt{\frac{c}{2 N}}\left(a_{2}+a_{2}^{*}\right)(\tau, \lambda), \quad p_{\bar{\phi}}(\tau, \lambda)=-i \sqrt{\frac{N}{2 c}}\left(a_{2}-a_{2}^{*}\right)(\tau, \lambda), \\
& a_{1}(\tau, \lambda)=\left(\sqrt{\frac{N}{2 c}} q_{\bar{\theta}}+i \sqrt{\frac{c}{2 N}} p_{\bar{\theta}}\right)(\tau, \lambda), \quad a_{2}(\tau, \lambda)=\left(\sqrt{\frac{N}{2 c}} q_{\bar{\phi}}+i \sqrt{\frac{c}{2 N}} p_{\bar{\phi}}\right)(\tau, \lambda) .
\end{aligned}
$$

In terms of these variables the invariant mass and the rest spin have the following expression

$$
\begin{aligned}
M= & \int_{o}^{\pi} d \lambda \sqrt{-N^{2}+A^{2}(\tau, \lambda)+B^{2}(\tau, \lambda)}, \\
A(\tau, \lambda)= & \frac{a_{1} \partial_{\lambda} a_{1}^{+}-a_{1}^{+} \partial_{\lambda} a_{1}}{2 \sqrt{2 a_{1}^{+} a_{1}}}(\tau, \lambda) \int_{o}^{\lambda} d \lambda_{1}\left(a_{1} \partial_{\lambda} a_{1}^{+}-a_{1}^{+} \partial_{\lambda} a_{1}+a_{2} \partial_{\lambda} a_{2}^{+}-a_{2}^{+} \partial_{\lambda} a_{2}\right)\left(\tau, \lambda_{1}\right)+ \\
+ & {\left[a_{1} \partial_{\lambda} a_{1}^{+}+a_{1}^{+} \partial_{\lambda} a_{1}-\frac{1}{2}\left(a_{2} \partial_{\lambda} a_{2}^{+}-a_{2}^{+} \partial_{\lambda} a_{2}\right) \frac{a_{1}+a_{1}^{+}}{a_{1}-a_{1}^{+}}\right](\tau, \lambda), } \\
B(\tau, \lambda)= & \frac{i}{4}\left(\frac{a_{1}-a_{1}^{+}}{\sqrt{a_{1}^{+} a_{1}}} \frac{a_{2} \partial_{\lambda} a_{2}^{+}-a_{2}^{+} \partial_{\lambda} a_{2}}{\sqrt{2 a_{2}^{+} a_{2}}}\right)(\tau, \lambda) \\
& \int_{o}^{\lambda} d \lambda_{1}\left(a_{1} \partial_{\lambda} a_{1}^{+}-a_{1}^{+} \partial_{\lambda} a_{1}+a_{2} \partial_{\lambda} a_{2}^{+}-a_{2}^{+} \partial_{\lambda} a_{2}\right)\left(\tau, \lambda_{1}\right)+ \\
+ & {\left[2 i \frac{\sqrt{a_{1}^{+} a_{1}}}{a_{1}-a_{1}^{+}}\left(a_{1} \partial_{\lambda} a_{1}^{+}+a_{1}^{+} \partial_{\lambda} a_{1}\right)-2 i \frac{a_{1}^{+}+a_{1}}{\left(a_{1}-a_{1}^{+}\right) 2} \frac{a_{2}^{+} a_{2}}{\sqrt{a_{1}^{+} a_{1}}}\left(a_{1} \partial_{\lambda} a_{1}^{+}-a_{1}^{+} \partial_{\lambda} a_{1}\right)\right.} \\
+ & \left.i \frac{a_{1}+a_{1}^{+}}{2} \frac{\sqrt{a_{1}^{+} a_{1}}}{\sqrt{2 a_{2}^{+} a_{2}}}\left(a_{2} \partial_{\lambda} a_{2}^{+}-a_{2}^{+} \partial_{\lambda} a_{2}\right)\right](\tau, \lambda),
\end{aligned}
$$




$$
\begin{aligned}
\vec{S} & =\frac{1}{2} \int_{-\pi}^{\pi} d \lambda \vec{S}(\tau, \lambda), \\
S^{1}(\tau, \lambda) & =-\frac{i}{2} \frac{\left(a_{1}^{*}-a_{1}\right)\left(a_{2}^{*}-a_{2}\right) a_{1}^{*} a_{1}-\left(a_{1}^{*}+a_{1}\right)\left(a_{2}^{*}+a_{2}\right) a_{2}^{*} a_{2}}{\left(a_{1}^{*}-a_{1}\right) \sqrt{a_{2}^{*} a_{2}}}(\tau, \lambda), \\
S^{2}(\tau, \lambda) & =+\frac{i}{2} \frac{\left(a_{1}^{*}-a_{1}\right)\left(a_{2}^{*}+a_{2}\right) a_{1}^{*} a_{1}+\left(a_{1}^{*}+a_{1}\right)\left(a_{2}^{*}-a_{2}\right) a_{2}^{*} a_{2}}{\left(a_{1}^{*}-a_{1}\right) \sqrt{a_{2}^{*} a_{2}}}(\tau, \lambda), \\
S^{3}(\tau, \lambda) & =-\left(a_{2}^{*} a_{2}\right)(\tau, \lambda) .
\end{aligned}
$$

This representation is well defined if we have $a_{1}^{*}(\tau, \lambda)-a_{1}(\tau, \lambda) \neq 0$ for every $\lambda$. 


\section{CONCLUSIONS}

In this paper we have developed the rest-frame instant form of the open Nambu string (the closed one could be treated in the same way). The string can be described as a frozen decoupled non-local canonical non-covariant Newton-Wigner center of mass $(\vec{z}, \vec{h})$ plus a canonical basis of Wigner-covariant relative variables restricted by transversality first-class constraints implying that only modes orthogonal to the string are physical. The NewtonWigner center of mass carries a universal external realization of the Poincare' algebra, which depends on the string relative variables only through the invariant mass $M$ and the rest-spin $\overrightarrow{\vec{S}}$.

We have found two canonical bases of gauge invariant Dirac observables by using FrenetSerret geometrical methods and by Abelianizing the transversality constraints.

The first canonical basis of Dirac observables is of the type of action-angle variables and it can be reformulated in terms of two sets of transverse oscillators, giving rise to a non-linear realization of the $\mathrm{O}(3)$ algebra of the rest spin (like in a $\sigma$-model).

The second one, where the Dirac observables are Lorentz scalars, is only implicitly known. In it the Dirac observables are the Frenet-Serret curvature and torsion of the string plus the conjugate momenta.

Let us end with some comments on canonical quantization. In the usual approach one first quantizes and then makes the reduction to the physical states by imposing the quantum constraints expressed in terms of Fock operators satisfying the Virasoro algebra. This is done in the light-cone gauge and only in the critical dimension dimension $d=26$ does one get a quantum realization of the Poincare' algebra. There is a tachyon and a naive treatment of the relativistic center of mass. The papers in Refs. [8, 11, 24] (and [12] for the closed string) were attempts to find Wigner-covariant classical oscillators for this approach.

Another stimulus to develop the rest-frame instant form came from Ref.[25], were the string was studied with the techniques of the inverse scattering methods (Lax pairs). This allowed one to find a recursive relation for building action-angle variables (with the action ones globally defined) and then special oscillators giving rise to rising Regge trajectories and without tachyons. The final statement of this approach was that a certain family of motions of the string without cusp singularities ("smooth strings") can be quantized in dimension $d=4$. However one has still to solve the constraints at the quantum level.

Instead in Refs.[26-28] there is the definition of an infinite-dimensional algebra of gaugeinvariant conserved charges (including the Poincare' generators), the so-called PohlmeyerRehren constants of the motion. Again the center of mass is treated in a naive way. These charges are quantized in dimension $d=4$ with methods of algebraic quantization by means of a deformation of the algebra which is supposed to be without anomalies (a final proof is still lacking). See the review in Ref.[29], where a different quantization in the framework of loop quantum gravity is tried. However, not withstanding all the technical developments, it is shown in Ref.[30] that this quantization is not equivalent to the standard canonical quantization with a Fock space: in every dimension there are anomalies if the invariant charges are quantized in a Fock space.

The classical results of this paper may be relevant for the approach "first reduce and then quantize", which generically is inequivalent to the standard one. 
The open problem with our approach concerns the quantization method. Since actionangle variables cannot be quantized consistently due to the angles [31], one should quantize the non-linear realization of the $\mathrm{O}(3)$ algebra plus the invariant mass. Due to the complexity of the non-linear realization of $\mathrm{O}(3)$, this is a highly non-trivial problem and any result would be welcome (for instance a statement on the existence of critical dimensions). As shown in Ref.[32] the canonical quantization of the frozen decoupled canonical Newton-Wigner center of mass can be done in a way which avoids the causality problems connected with the Hegerfeldt theorem (instantaneous spreading of wave packets) and allows one to define an intrinsic Wigner-covariant inner space of rest relative variables. Moreover the non-locality of the non-covariant center of mass makes it not observable (like the wave function of the universe!) and this makes its universal non-covariance irrelevant. If $M$ and $\vec{S}$ can become self-adjoint operators with the spin operator satisfying the $\mathrm{O}(3)$ algebra and commuting with the invariant mass operator then there is no problem in quantizing the external Poincare' algebra.

The same scheme should be used if we could express the invariant mass and the rest spin in terms of the FS curvature and torsion and of the conjugate momenta. However, since we have $\rho_{1}(\tau, \lambda) \geq 0$ and $\rho_{2}(\tau, \lambda) \geq 0$, these variables are like radial variables and in trying to quantize them one should use the non-canonical basis $r$ and $r p_{r}$, the only one which would give meaningful commutators due to $r \geq 0$. 


\section{Appendix A: Distributions for the Nambu String}

We collect here the definitions of the most important distributions used in this paper, which were given in the Appendix of Ref.[11]. Many of them where already present in Ref.[19].

The even and odd $\delta$-functions with $2 \pi$-periodicity are respectively

$$
\begin{aligned}
\Delta_{+}(\sigma, \bar{\sigma}) & =\frac{1}{2 \pi} \sum_{n=-\infty}^{\infty}\left[e^{i n(\sigma-\bar{\sigma})}+e^{i n(\sigma+\bar{\sigma})}\right]=\sum_{n=-\infty}^{\infty}[\delta(\sigma-\bar{\sigma}+2 n \pi)+\delta(\sigma+\bar{\sigma}+2 n \pi)]= \\
& =\partial_{\sigma} \sum_{n=-\infty}^{\infty}[\theta(\sigma-\bar{\sigma}+2 n \pi)+\theta(\sigma+\bar{\sigma}+2 n \pi)]=\frac{1}{\pi}+\partial_{\sigma} \Sigma_{+}(\sigma, \bar{\sigma}),
\end{aligned}
$$

and

$$
\begin{aligned}
\Delta_{-}(\sigma, \bar{\sigma}) & =\frac{1}{2 \pi} \sum_{n=-\infty}^{\infty}\left[e^{i n(\sigma-\bar{\sigma})}-e^{i n(\sigma+\bar{\sigma})}\right]=\sum_{n=-\infty}^{\infty}[\delta(\sigma-\bar{\sigma}+2 n \pi)-\delta(\sigma+\bar{\sigma}+2 n \pi)]= \\
& =\partial_{\sigma} \sum_{n=-\infty}^{\infty}[\theta(\sigma-\bar{\sigma}+2 n \pi)-\theta(\sigma+\bar{\sigma}+2 n \pi)]=\partial_{\sigma} \Sigma_{-}(\sigma, \bar{\sigma})=-\partial_{\bar{\sigma}} \Sigma_{+}(\sigma, \bar{\sigma}),
\end{aligned}
$$

where $\theta(\sigma)$ is the step function $(=1$ for $\sigma>0 ;=0$ for $\sigma<0 ; \theta(\sigma)+\theta(-\sigma)=1)$ and $\delta(\sigma)$ $=\partial_{\sigma} \theta(\sigma)$ is the standard $\delta$-function. The sign-function is $\varepsilon(\sigma)=\theta(\sigma)-\theta(-\sigma)(=1$ for $\sigma>0 ;=-1$ for $\sigma<0 ; \varepsilon(\sigma)+\varepsilon(-\sigma)=0)$.

The delta functions $\Delta_{ \pm}$have the following properties

$$
\begin{aligned}
& \Delta_{+}\left(\lambda, \lambda^{\prime}\right)=\Delta_{+}\left(-\lambda, \lambda^{\prime}\right)=\Delta_{+}\left(\lambda^{\prime}, \lambda\right)=\Delta_{+}\left(\lambda+2 n \pi, \lambda^{\prime}\right), \\
& \Delta_{-}\left(\lambda, \lambda^{\prime}\right)=-\Delta_{-}\left(-\lambda, \lambda^{\prime}\right)=\Delta_{-}\left(\lambda^{\prime}, \lambda\right)=\Delta_{-}\left(\lambda+2 n \pi, \lambda^{\prime}\right), \\
& \Delta_{ \pm}^{\prime}\left(\lambda, \lambda^{\prime}\right)=\frac{\partial}{\partial \lambda} \Delta_{ \pm}\left(\lambda, \lambda^{\prime}\right)=-\frac{\partial}{\partial \lambda^{\prime}} \Delta_{\mp}\left(\lambda, \lambda^{\prime}\right), \\
& \int_{-\pi}^{\pi} d \lambda^{\prime} f\left(\lambda^{\prime}\right) \Delta_{ \pm}\left(\lambda^{\prime}, \lambda\right)=f(\lambda) \pm f(-\lambda) .
\end{aligned}
$$

We give some formulas for the distributions $\Delta_{ \pm}\left(\sigma, \sigma^{\prime}\right)$, used in the evaluation of some Poisson brackets. If $f(x)$ and $g(x)$ are periodic functions, with period $2 \pi$, and with definite parity given by $P_{f}= \pm 1$, if $f(x)= \pm f(-x)$, where, of course, $P_{f^{\prime}}=-P_{f}$, we have the following identities 


$$
\begin{aligned}
& f(x) g(y) \Delta_{ \pm}^{\prime}(x, y)=f(y) g(y) \Delta_{ \pm P_{f}}^{\prime}(x, y)-f^{\prime}(x) g(x) \Delta_{ \pm P_{g}}(x, y), \\
& f(x) g(y) \Delta_{+}^{\prime}(x, y) \pm f(y) g(x) \Delta_{-}^{\prime}(x, y)= \pm f(x) g(x) \Delta_{-P_{f}}^{\prime}(x, y)+ \\
& +f(y) g(y) \Delta_{+P_{f}}^{\prime}(x, y)-f^{\prime}(x) g(x)\left[\Delta_{+P_{g}}(x, y) \mp \Delta_{-P_{f}}(x, y)\right], \\
& {[f(x) g(y)-f(y) g(x)] \Delta_{ \pm}^{\prime}(x, y)=[f(y) g(y)-f(x) g(x)] \Delta_{ \pm P_{f}}^{\prime}(x, y)-} \\
& -f^{\prime}(x) g(x)\left[\Delta_{ \pm P_{g}}(x, y)+\Delta_{ \pm P_{f}}(x, y)\right], \\
& {[f(x) g(y)+f(y) g(x)] \Delta_{ \pm}^{\prime}(x, y)=[f(y) g(y)+f(x) g(x)] \Delta_{ \pm P_{f}}^{\prime}(x, y)-} \\
& -f^{\prime}(x) g(x)\left[\Delta_{ \pm P_{g}}(x, y)-\Delta_{ \pm P_{f}}(x, y)\right] .
\end{aligned}
$$


[1] L. Lusanna, The $N$ - and 1-Time Classical Descriptions of N-Body Relativistic Kinematics and the Electromagnetic Interaction, Int. J. Mod. Phys. A12, 645 (1997).

L.Lusanna, The Chrono-Geometrical Structure of Special and General Relativity: A ReVisitation of Canonical Geometrodynamics, lectures at 42nd Karpacz Winter School of Theoretical Physics: Current Mathematical Topics in Gravitation and Cosmology, Ladek, Poland, 6-11 Feb 2006, Int.J.Geom.Methods in Mod.Phys. 4, 79 (2007). (gr-qc/0604120).

[2] L.Lusanna, Towards Relativistic Atomic Physics and Post-Minkowskian Gravitational Waves, talk at the Workshop on Gravitational Waves Detection with Atom Interferometry, held in Firenze at the Galileo Galilei Institute for Theoretical Physics, February 23-24, 2009 (0908.0209).

L.Lusanna, Post-Minkowskian Gravity: Dark Matter as a Relativistic Inertial Effect?, Talk at the 1st Mediterranean Conference in Classical and Quantum Gravity, held in the Orthodox Academy of Crete in Kolymbari (Greece) from Monday, September 14th to Friday, September 18th, 2009 (0912.2935).

[3] D.Alba and L.Lusanna, Charged Particles and the Electro-Magnetic Field in Non-Inertial Frames: I. Admissible 3+1 Splittings of Minkowski Spacetime and the Non-Inertial Rest Frames (0908.0213), Int.J.Geom. Methods in Physics, 7, 33 (2010); II. Applications: Rotating Frames, Sagnac Effect, Faraday Rotation, Wrap-up Effect (0908.0215), Int.J.Geom. Methods in Physics, 7, 185 (2010).

[4] D.Alba, H.W.Crater and L.Lusanna, Towards Relativistic Atom Physics. I. The Rest-Frame Instant Form of Dynamics and a Canonical Transformation for a system of Charged Particles plus the Electro-Magnetic Field, to appear in Canad.J.Phys. (0806.2383).

[5] D.Alba, H.W.Crater and L.Lusanna, Towards Relativistic Atom Physics. II. Collective and Relative Relativistic Variables for a System of Charged Particles plus the Electro-Magnetic Field, to appear in Canad.J.Phys. (0811.0715).

[6] D.Alba, H.W.Crater and L.Lusanna, Hamiltonian Relativistic Two-Body Problem: Center of Mass and Orbit Reconstruction, J.Phys. A40, 9585 (2007) (gr-qc/0610200).

H.Crater and L.Lusanna, The Rest-Frame Darwin Potential from the Lienard-Wiechert Solution in the Radiation Gauge, Ann.Phys.(N.Y.) 289, 87 (2001)(hep-th/0001046).

D.Alba, H.Crater and L.Lusanna, The Semiclassical Relativistic Darwin Potential for Spinning Particles in the Rest-Frame Instant Form: Two-Body Bound States with Spin 1/2 Constituents, Int.J.Mod.Phys. A16, 3365 (2001) (hep-th/0103109).

[7] Y.Nambu, Quark Model and the Factorization of Veneziano Amplitude, Proc.Int.Conf. on Symmetries and Quark Models, 1969, Wayne State Univ. (Gordon and Breach, NewYork, 1970).

T.Goto, Relativistic Quantum Mechanics of One-Dimensional Mechanical Continuum and Subsidiary Condition of Dual Resonance Model, Prog.Theor.Phys. 46, 1560 (1971).

J.Scherk, An Introduction to the Theory of Dual Models and Strings, Rev.Mod.Phys. 47, 123 (1975).

J.H.Schwarz, Superstring Theory, Phys.Rep. 89, 223 (1982).

[8] F.Colomo, G.Longhi and L.Lusanna, Classical Canonical Observables of the Nambu String, Mod.Phys.Lett. A5, 17 (1990).

[9] H.Leutwyler and J.Stern, Relativistic Dynamics on a Null Plane, Ann.Phys. (N.Y.) 112, 94 
(1978).

[10] F.Colomo, G.Longhi and L.Lusanna, Classical Solutions of the Many-Time Functional Equations of Motion of the Nambu String, Int.J.Mod.Phys. A5, 3347 (1990).

[11] F.Colomo and L.Lusanna, Wigner Theory of the Nambu String: I. The Open String, Int.J.Mod.Phys. A7, 1705 (1992).

[12] F.Colomo and 1.Lusanna, Wigner Theory of the Nambu String: I. The Closed String, Int.J.Mod.Phys. A7, 4107 (1992).

[13] D.C.Salisbury and K.Sundermeyer, Stationary Properties of Nambu Strings, Nucl.Phys. B191, 250 (1981).

[14] D.G.Boulware and E.T.Newman, The Geometry of Open Bosonic String, Phys.Letters, B174, 378 (1986).

[15] X.Artru, Classical String Phenomenology. 1. How Strings Work, Phys.Rev. 97, 147 (1983).

[16] E.Del Giudice,P.Di Vecchia and S.Fubini, General Properties of the Dual Resonance Model, Ann.Phys.(NY) 70, 378 (1972).

[17] S.Fubini, D.Gordon and G.Veneziano, A General Treatment of Factorization in Dual Resonance Models, Phys. Letters 29B, 679 (1969).

[18] B.M.Barbashov and V.V.Nesterenko, Dynamics of a Relativistic String, Sov.J.Part.Phys. 9, 391 (1978).

[19] A.J.Hanson, T.Regge and C.Teitelboim, Constrained Hamiltonian Systems, in Contributi del Centro Linceo Interdisciplinare di Scienze Matematiche, Fisiche e loro Applicazioni, n.22 (Accademia Nazionale dei Lincei, Roma, 1975).

[20] A.Patrascioiu, Quantum Dynamics of a Massless Relativistic String, Lett. Nuovo Cimento 10, 676 (1974); Quantum Dynamics of a Massless Relativistic String. 2, Nucl.Phys. B81, 525 (1974).

[21] R.Benguria, P.Cordero and C.Teitelboim, Aspects of the Hamiltonian Dynamics of Interacting Gravitational Gauge and Higgs Fields with Applications to Spherical Symmetry, Nucl.Phys. $\underline{B 122}, 61$ (1977).

[22] P.J.Steinhardt, Problems of Quantization in the Infinite Momentum Frame, Ann.Phys. (N.Y.) $\underline{128}, 425(1980)$.

[23] M.Spivak, A Comprehensive Introduction in Differential Geometry, Vol. IV (Houston, Publish or Perish Inc., 1979, second edition).

[24] L.Lusanna, Dirac's Observable: From Particles to String and Fields, in Proc. of the Int. Symp. on Extended Objects and Bound Systems, Karuizawa, Japan, Mar 19-21, 1992, eds. O.Hara, S.Ishida and S.Naka (World Scientific, Singapore, 1993).

[25] G.P.Pron'ko, Hamiltonian Theory of the Relativistic String, Rev.Math.Phys. 2,355 (1991) and references therein.

[26] K.Pohlmeyer, A Group Theoretical Approach to the Quantization of the Free Relativistic Closed String, Phys.Lett. 119B, 100 (1982); The algebra formed by the Charges of the NambuGoto String: Casimir Elements, Commun.Math.Phys. 114, 351 (1988); The Nambu-Goto Theory of the Closed Bosonic Strings Moving in (1+3)-Dimensional Minkowski Space, Annalen Phys. 8, 19 (1999).

K.Pohlmeyer and K.H.Rehren, Algebraic Properties of the Invariant Charges of the NambuGoto Theory, Commun.Math.Phys. 105, 593 (1986); The Algebra formed by the Charges of the Nambu-Goto Theory: Identification of a Maximal Abelian Subalgebra, Commun.Math.Phys. 114, 55 (1988); The Algebra formed by the Charges of the Nambu-Goto Theory: Their Geometric Origin and their Completeness, Commun.Math.Phys. 114, 177 (1988). 
K.Pohlmeyer and M.Trunk, The Invariant Charges of the Nambu-Goto Theory: Quantization of Non-Additive Composition Laws, Int.J.Mod.Phys. A19, 115 (2004)(hep-th/0206061).

[27] C.Meusburger and K.H.Rehren, Algebraic Quantization of the Closed Bosonic String, Commun.Math.Phys. 237, 69 (2003) (math-ph/0202041).

[28] G.Handrich, Lorentz Covariance of the Quantum Algebra of Observables: Nambu-Goto Strings in 3+1 Dimensions, Int.J.Mod.Phys. A17, 2331 (2002).

[29] T.Thiemann, THE LQG - String: Loop Quantum Gravity Quantization of String Theory. I. Flat Target Space, Clas. Quantum Grav. 23, 1923 (2006) (hep-th/0401172).

[30] D.Bahns, The Invariant Charges of the Nambu-Goto String and Canonical Quantization, J.Math.Phys. 45, 4640 (2004) (hep-th/0403108).

[31] R.Lynch, The Quantum Phase Problem: A Critical Review, Phys.Rep. 256, 367 (1995).

Eds. W.P.Schleich and S.M.Barnett, Quantum Phase and Phase Dependent Measurements, Physica Scripta vol. T48, (1993).

[32] D.Alba, H.W.Crater and L.Lusanna, Relativistic Quantum Mechanics and Relativistic Entanglement in the Rest-Frame Instant Form of Dynamics, (arXiv 0907.1816). 